\title{
THE MAGELLANIC QUASARS SURVEY. III. SPECTROSCOPIC CONFIRMATION OF 758 ACTIVE GALACTIC NUCLEI BEHIND THE MAGELLANIC CLOUDS
}

\author{
Szymon KozŁowski ${ }^{1,9}$, Christopher A. Onken ${ }^{2}$, Christopher S. KochaneK $^{3,4}$, Andrzej Udalski ${ }^{1,9}$, \\ AND \\ M. K. Szymański ${ }^{1}$, M. Kubiak ${ }^{1}$, G. Pietrzyński ${ }^{1,5}$, I. Soszyński ${ }^{1}$, Ł. Wyrzykowski ${ }^{1,6}$, K. UlaczyK ${ }^{1}$, \\ R. Poleski ${ }^{1,3}$, P. Pietrukowicz ${ }^{1}$, J. SKOWron $^{1}$ \\ (The OGLE COLlaboration) \\ AND \\ M. MeiXner ${ }^{7}$, A. Z. Bonanos ${ }^{8}$ \\ ${ }^{1}$ Warsaw University Observatory, Al. Ujazdowskie 4, 00-478 Warszawa, Poland; simkoz@ astrouw.edu.pl \\ ${ }^{2}$ Research School of Astronomy and Astrophysics, The Australian National University, Canberra 2611, Australia; onken@ mso.anu.edu.au \\ ${ }^{3}$ Department of Astronomy, The Ohio State University, 140 West 18th Avenue, Columbus, OH 43210, USA; ckochanek@astronomy.ohio-state.edu \\ ${ }^{4}$ The Center for Cosmology and Astroparticle Physics, The Ohio State University, 191 West Woodruff Avenue, Columbus, OH 43210, USA \\ ${ }_{6}^{5}$ Departamento de Astronomia, Universidad de Concepción, Casilla 160-C, Concepción, Chile \\ ${ }^{6}$ Institute of Astronomy, University of Cambridge, Madingley Road, Cambridge CB3 0HA, UK \\ ${ }^{7}$ Space Telescope Science Institute, 3700 San Martin Drive, Baltimore, MD 21218, USA \\ ${ }^{8}$ National Observatory of Athens, Institute of Astronomy, Astrophysics, Space Applications and \\ Remote Sensing, I. Metaxa and Vas. Pavlou St., Palaia Penteli, 15236 Athens, Greece \\ Received 2013 May 29; accepted 2013 July 24; published 2013 September 11
}

\begin{abstract}
The Magellanic Quasars Survey (MQS) has now increased the number of quasars known behind the Magellanic Clouds by almost an order of magnitude. All survey fields in the Large Magellanic Cloud (LMC) and 70\% of those in the Small Magellanic Cloud (SMC) have been observed. The targets were selected from the third phase of the Optical Gravitational Lensing Experiment (OGLE-III) based on their optical variability, mid-IR, and/or X-ray properties. We spectroscopically confirmed 758 quasars (565 in the LMC and 193 in the SMC) behind the clouds, of which $94 \%$ (527 in the LMC and 186 in the SMC) are newly identified. The MQS quasars have long-term (12 yr and growing for OGLE), high-cadence light curves, enabling unprecedented variability studies of quasars. The MQS quasars also provide a dense reference grid for measuring both the internal and bulk proper motions of the clouds, and 50 quasars are bright enough $(I \lesssim 18 \mathrm{mag}$ ) for absorption studies of the interstellar/intergalactic medium of the clouds.
\end{abstract}

Key words: galaxies: active - Magellanic Clouds - quasars: general

Online-only material: machine-readable tables

\section{INTRODUCTION}

The Magellanic Clouds (MCs) are the nearest well-resolved dwarf galaxies and for decades have been an ideal playground for testing stellar and galaxy evolution theories (e.g., Groenewegen \& de Jong 1993; Meixner et al. 2006; van der Marel et al. 2002; Kallivayalil et al. 2013; van der Marel \& Kallivayalil 2013), establishing the stellar initial mass function (e.g., Humphreys \& McElroy 1984), investigating metallicity (e.g., Massey et al. 1995), studying dust properties (e.g., Weingartner \& Draine 2001), and testing the (ultimately falsified) hypothesis of dark matter being comprised of non-luminous compact objects (e.g., Alcock et al. 2000; Tisserand et al. 2007; Wyrzykowski et al. 2011). Such intensively monitored areas are also ideal for finding and studying variable objects. For example, the third phase of the Optical Gravitational Lensing Experiment (OGLE) produced the OGLE-III Catalog of Variable Stars, the largest uniform catalog of variable stars in the MCs with over 175,000 objects (e.g., Soszyński et al. 2008, 2009a, 2009b, 2010). The proximity of the MCs make them well suited to testing and calibrating cosmological distance indicators (see Alves 2004 for a review), such as eclipsing binaries

\footnotetext{
9 The OGLE Collaboration.
}

(e.g., Bonanos et al. 2011; Pietrzyński et al. 2013), the tip of the red giant branch (Cioni et al. 2000; Udalski 2000a), Cepheids (Feast 1999; Gieren et al. 1998; Soszyński et al. 2008), RR Lyrae (Udalski 2000a), cluster main-sequence fitting (Schommer et al. 1984), or red clump stars (Udalski 2000b; Alves et al. 2002).

There are, however, more uses for such a huge database of photometric records collected in this region of the sky. With little Galactic or MC extinction, it is in principle straightforward to find supernovae (e.g., Kozłowski et al. 2013) and active galactic nuclei $\left(\mathrm{AGNs}^{10}\right.$ ) behind the MCs. The challenge, of course, is that while there are $\sim 25$ quasars $\mathrm{deg}^{-2}$ with $I<20 \mathrm{mag}$, there are over $10^{6}$ stars $\mathrm{deg}^{-2}$ in the MCs. With so many stars of different types, optical color selection methods have too high a false positive rate. Wide area X-ray surveys suffer both from contamination by accreting sources in the MCs and low $(\sim$ arcmin) resolution that makes it difficult to correctly identify the optical counterpart. Early searches based on variability lacked robust, quantitative means of distinguishing the aperiodic variability of quasars and stars. Despite these difficulties, some $\sim 80$ quasars had been discovered behind the MCs as of 2009 (Schmidtke et al. 1994; Dobrzycki et al. 2002, 2003a, 2003b;

\footnotetext{
10 The terms AGNs, quasars, and QSOs will be used interchangeably
} throughout this paper. 
Geha et al. 2003; Dobrzycki et al. 2005) and they were a crucial part of the projects to accurately measure the proper motions of the MCs (Kallivayalil et al. 2006a, 2006b; Piatek et al. 2008; Kallivayalil et al. 2013). Now, however, these projects are limited by the lack of a denser reference grid that can be used to better measure and separate the internal and bulk motions of the MCs (e.g., Kallivayalil et al. 2013). However, expanding the quasar sample by an order of magnitude is probably not feasible using the approaches of these earlier searches.

The first major improvements became possible with the advent of the Spitzer Space Telescope (Werner et al. 2004), which enabled mid-IR surveys of the MCs such as the Surveying the Agents of Galaxy Evolution (SAGE; Meixner et al. 2006), Surveying the Agents of Galaxy Evolution-Small Magellanic Cloud (SAGE-SMC; Gordon et al. 2011), and the Spitzer Survey of the Small Magellanic Cloud $\left(\mathrm{S}^{3} \mathrm{MC}\right.$; Bolatto et al. 2007 ) projects. At the same time, it was realized in extragalactic surveys that mid-IR colors were a powerful means of distinguishing stars, galaxies and AGNs - in particular, almost all red mid-IR sources are quasars because they have a flatter spectral energy distribution than the Rayleigh-Jeans law that (roughly) characterizes stars and low redshift galaxies (Lacy et al. 2004; Stern et al. 2005). In Kozłowski \& Kochanek (2009), we showed that this fact was also true in the dense stellar fields of the MCs, particularly with the addition of limits on the OGLE-III (Udalski et al. 2008a, 2008b, 2008c) I-band-to-mid-IR colors, albeit with some additional contamination from the higher abundances of dusty stars and young stellar objects (YSOs).

At the same time, Kelly et al. (2009) proposed that quasar light curves were well modeled by a stochastic process, the damped random walk (DRW), which is characterized by an exponential covariance matrix defined by an asymptotic variance $\sigma$ and a timescale $\tau$. In Kozłowski et al. (2010), we showed that the mid-IR quasar candidates from Kozłowski \& Kochanek (2009) largely lay in a different region of $\sigma / \tau$ parameter space than variable stars, thus providing a robust, quantitative means of selecting quasars on the basis of variability. Kozłowski et al. (2010) also (re)introduced a more statistically powerful method of estimating the DRW parameters than was used by Kelly et al. (2009), based on the methods previously discussed by Press et al. (1992) and Rybicki \& Press (1992, 1995). The power of the method used by Kozłowski et al. (2010) was then confirmed by MacLeod et al. (2010) using the Sloan Digital Sky Survey (SDSS) variability data for $\sim 9000$ spectroscopically confirmed quasars in SDSS Stripe 82 AGNs with extensions by MacLeod et al. (2011) and Butler \& Bloom (2011). Other recent studies of selecting quasars on the basis of variability are detailed in Eyer (2002), Schmidt et al. (2010), Palanque-Delabrouille et al. (2011), Kim et al. (2012), and Pichara et al. (2012).

Kelly et al. (2009) and MacLeod et al. (2010) further showed that the DRW parameters are correlated with wavelength, luminosity, and black hole mass, and MacLeod et al. (2012) showed that the DRW model can fully explain the variability statistics of ensembles of quasars. Zu et al. (2013) and Andrae et al. (2013) showed that the DRW model is a better model of stochastic process for quasar light curves on timescales of days to years than many simple variants, although Mushotzky et al. (2011) found that four Kepler-monitored AGNs potentially have power spectra steepening on very short timescales. The DRW model then provides a very well-defined means of carrying out the interpolations needed when cross-correlating light curves, as shown in the reanalysis of quasar reverberation mapping light curves by $\mathrm{Zu}$ et al. (2011), and it could play a similar role in measuring the time delays of lensed quasars (e.g., Press et al. 1992, Hojjati et al. 2013, who used a stochastic model but not the DRW model).

A fundamental problem with this renaissance in quasar variability studies is that the SDSS Stripe 82 light curves are not, in fact, very good, comprising only 60 epochs for each quasar with large temporal gaps. In fact, the quasars with the best, densely sampled, long-term light curves are the quasars behind the MCs, because they have been almost continuously monitored by microlensing projects for over a decade. The typical quasar has $\sim 500 \mathrm{I}$-band and $\sim 50 \mathrm{~V}$-band epochs from OGLE-III (years 2001-2009) and $\sim 500$ I-band and $\sim 100$ $V$-band epochs from OGLE-IV (years 2010-2013) and the light curves continue to be extended. (The MACHO data span $7 \mathrm{yr}$ with about 1000 data points in both "standardizable" $V$ - and $R$-bands.) The superiority of these light curves is likely to remain the case until $10 \mathrm{yr}$ after the advent of LSST (Ivezic et al. 2008), although Pan-STARRS (Kaiser et al. 2002) will provide larger numbers of more sparsely sampled, multi-color light curves. As noted earlier, denser networks of quasars behind the MCs are also needed for improved proper motion measurements.

We started the Magellanic Quasars Survey (MQS) in 2009 to greatly expand the number of AGNs behind the MCs using the $3.9 \mathrm{~m}$ Anglo-Australian Telescope (AAT) and the AAOmega spectrograph. AAOmega allows multi-object spectroscopy of 400 targets within a $3 \mathrm{deg}^{2}$ field of view (e.g., Sharp et al. 2006). Although the runs were plagued by bad weather, we reported the discovery of 29 new AGNs behind the SMC (doubling their number) in Kozłowski et al. (2011, hereafter Paper I) and the discovery of 144 new AGNs behind the Large Magellanic Cloud (LMC) (quadrupling their number) in Kozłowski et al. (2012, hereafter Paper II). Here, we bring the present phase of the MQS (spectroscopic confirmations) to a conclusion, where we have completed all 12 of the planned LMC fields and three out of five of the planned SMC fields (plus the pilot study from Paper I), confirming a total of 758 AGNs, which represents an increase in the number of known quasars behind the MCs by almost an order of magnitude. In Section 2, we describe the AGN selection procedures and in Section 3 we describe the data and their analysis. New AGNs are presented in Section 4 and the contaminating objects and objects with featureless spectra are described in Section 5. In Section 6, we discuss the relative detection efficiencies of our AGN selection methods. The paper is summarized in Section 7 where we outline future areas of exploration.

\section{AGN SELECTION PROCEDURES}

Our main driver for finding AGNs behind the MCs was to study their variability. We therefore limited our search to the well-monitored OGLE-III fields (Udalski et al. 2008c, 2008a, 2008b). We cross-matched the OGLE data with the SAGE, SAGE-SMC, and $\mathrm{S}^{3} \mathrm{MC}$ mid-IR data (Meixner et al. 2006; Gordon et al. 2011; Bolatto et al. 2007) and the ROSAT X-ray catalogs (Haberl \& Pietsch 1999; Haberl et al. 2000). The exact selection procedures are discussed in Paper II. For completeness, we briefly outline these procedures here.

Method 1. In the first method, we use the mid-IR/optical color-selected AGN candidates from Kozłowski \& Kochanek (2009), as illustrated in Figure 1. In the mid-IR color-color space, we defined a wedge following Stern et al. (2005) that we further subdivided into region $\mathrm{A}$, which should be free of low temperature (dusty) black bodies, and region B, which might contain these dusty objects. Then, in the mid-IR 

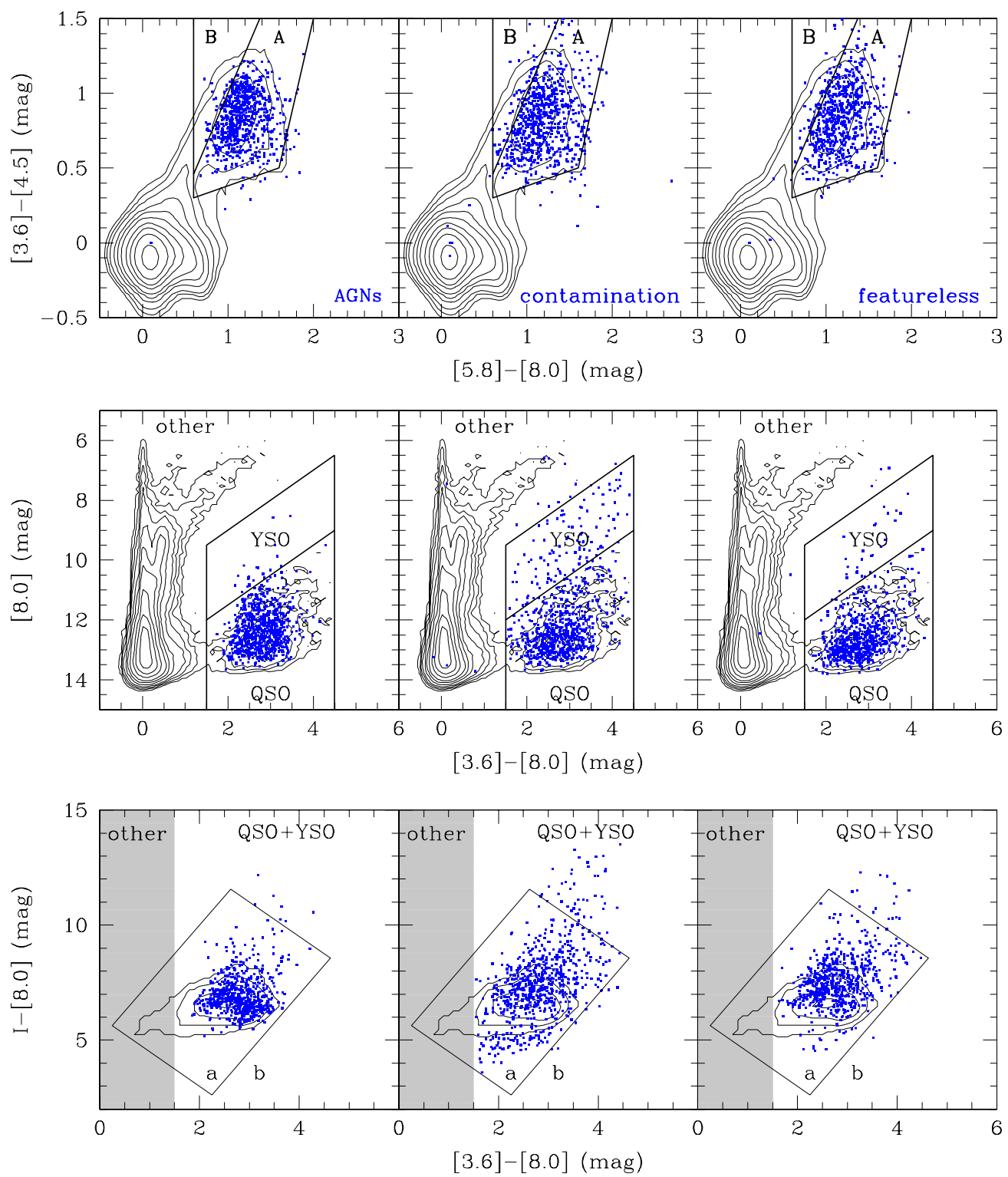

Figure 1. Mid-IR AGN selection criteria from Kozłowski \& Kochanek (2009). The points show the distribution of all MQS quasars (left panels), contaminating sources (middle panels) and featureless sources (right panels) and the labeled regions show the mid-IR/optical selection regions. Sources outside the selection regions did not meet the mid-IR selection criterion but were either variability- and/or X-ray-selected. The contours show the distribution of all SAGE (Meixner et al. 2006) sources in the LMC.

color-magnitude diagram, we defined a region likely to be heavily contaminated with YSOs and a region that should mostly contain quasars (QSOs). Finally, we defined objects with mid-IR-to-optical colors similar to AGNs as class "a" and those with other colors as class "b." Hence, each object has a classification such as QSO-Aa (most pure), QSO-Ab, ..., YSO-Ba, or YSO-Bb (most contaminated). Where there was no optical match (no detection in the OGLE or outside the OGLE fields), we assigned a classification of $(\mathrm{Q} / \mathrm{Y}) \mathrm{SO}-(\mathrm{A} / \mathrm{B}) 0$.

Method 2. Our second criterion was variability and our original intent was simply to use our criteria from Kozłowski et al. (2010). At the time, however, there was an unresolved problem in the long-term OGLE-III light curves involving inter-seasonal jumps in the photometry that then triggered large numbers of false positives when we simply fit the DRW model to all available light curves. Lacking time to resolve this problem for the full $\sim 42 \mathrm{deg}^{2}$ survey area, we largely adopted a variant of the Schmidt et al. (2010) structure function selection method to screen candidates because this selection was less sensitive to localized jumps. The resulting cuts were as follows (as in Paper II):

Cut 1. The average light curve magnitude is $16.0<I<$ $19.5 \mathrm{mag}$ for the LMC and $16.5<I<19.5 \mathrm{mag}$ for the SMC. The faint limit ensures that the data have a high enough signal-to-noise ratio $(\mathrm{S} / \mathrm{N})$ to provide a good light curve and the bright limit eliminates variable stars at fluxes where we have no significant expectation of finding a quasar given the survey area (see Kozłowski et al. 2010);

Cut 2. The light curve must be fit by some DRW model better than it is fit by white noise $\left(\ln L_{\text {best }}>\ln L_{\text {noise }}+2\right.$; Kozłowski et al. 2010). This cut essentially selects sources that are variable due to something other than noise;

Cut 3. We applied a very loose constraint on the DRW model timescale $(1<\log (\tau /$ days $)<5)$ and no constraint on $\hat{\sigma}$. The restrictions on $\tau$ removed $62 \%$ of the sources, mostly on short timescales. 
Cut 4. The slope of the light curve's structure function was broadly consistent with that of a quasar $(0.1<\gamma<0.9$; see Schmidt et al. 2010);

Cut 5. Finally, we also limited the associated $I$-band amplitude of the structure function to $A<0.4$ mag to remove high amplitude variable stars.

In an ideal world, we would have used a "cleaner" procedure so that our final discussion of the variability selection results would be simpler. On the other hand, we had no shortage of fibers, so there was no harm in using a rather broad definition for variability-selected candidates. In Section 6, we also comment on results when applying the original Kozłowski et al. (2010) variability criteria.

Method 3. Finally, we included variable objects with positions consistent with the location of any ROSAT X-ray source. Where there were multiple variable sources, the one closest to the X-ray position was included.

The AAT/AAOmega CONFIGURE software (Lewis et al. 2002; Miszalski et al. 2006) allows assigning priorities to targeted objects, where priority 1 is the lowest and priority 9 is the highest. We prioritized our candidates in the following way: priority 9 was assigned to objects selected by all three methods, priority 8 was assigned to objects meeting any two selection criteria, and priority 7 was assigned to objects selected by a single method. With the availability of fibers, we also included 931 stars (286 observed) that could potentially have been stripped from the 47 Tuc globular cluster (Lane et al. 2012). These stars should be easy to kinematically separate from SMC stars even with our relatively low spectral resolution. These stars were assigned priority 6 and their results will be presented elsewhere.

It is important to realize that we are not trying to produce a very high purity candidate sample because AAOmega has a significantly higher density of fibers $\left(400 / 3 \mathrm{deg}^{2} \approx 130 \mathrm{deg}^{-2}\right)$ than there are $I<20$ mag quasars $\left(\sim 25 \mathrm{deg}^{-2}\right)$ for which we are likely to measure redshifts given the exposure times and the backgrounds created by the high stellar density and large aperture fibers. Every fiber is ultimately assigned to something that might be a candidate (modulo the 47 Tuc stars), although many will be in low purity sub-samples (e.g., YSO-Bb) or so faint that we will only obtain a redshift if the source has sufficiently strong lines. Contamination is particularly severe for the very brightest (and rarest) quasars that both have best light curves and are the most useful for any absorption-line studies. By definition, only $1 / 4-1 / 3$ of our targets can be quasars bright enough to measure a redshift, which means we will also produce a large spectroscopic catalog of dusty or otherwise peculiar stars as a consequence. The stellar content of the MQS will be considered elsewhere.

\section{DATA}

The three selection criteria lead to samples of 2434 and 1447 candidates behind the OGLE-III regions of the LMC and SMC, respectively. For completeness, we include all observed MQS sources (candidates and confirmed AGNs) in our summary tables, but for discussions of efficiencies we exclude the sources from Paper I (where we used somewhat different selection methods) that did not pass our selection criteria from Section 2. The basic information on the fields is provided in Table 1 and their locations on the sky are shown in Figure 2. The target integration time was $1.5 \mathrm{hr}(3 \times 30$ minutes $)$ for 13 of the 15 completed fields (Table 1). Three of the fields from Paper II had shorter-than-desired exposure times, but we repeated one
Table 1

The MQS Observing Log

\begin{tabular}{lcccccc}
\hline \hline Field & R.A. & Decl. & $N_{\text {cand }}$ & $N_{\text {QSO }}$ & $\begin{array}{c}T_{\text {exp }} \\
\text { (hr) }\end{array}$ & Paper \\
& & & & & \\
\hline LMC1 & $04: 41: 43$ & $-69: 50: 29$ & 217 & 45 & 1.5 & III \\
LMC2 & $04: 43: 19$ & $-68: 19: 18$ & 193 & 71 & 1.5 & III \\
LMC3 & $04: 56: 52$ & $-67: 07: 48$ & 209 & 53 & 1.5 & III \\
LMC4a & $05: 00: 51$ & $-70: 27: 49$ & 221 & 41 & 0.4 & II \\
LMC4b & $05: 00: 51$ & $-70: 27: 49$ & 216 & 59 & 1.5 & III \\
LMC5 & $05: 01: 42$ & $-68: 49: 26$ & 265 & 75 & 1.5 & III \\
LMC6 & $05: 14: 27$ & $-67: 35: 00$ & 189 & 46 & 1.9 & III \\
LMC7 & $05: 19: 43$ & $-69: 31: 07$ & 307 & 36 & 1.5 & II \\
LMC8 & $05: 21: 54$ & $-71: 02: 48$ & 247 & 60 & 1.0 & II \\
LMC9 & $05: 32: 54$ & $-68: 31: 01$ & 220 & 61 & 1.5 & III \\
LMC10 & $05: 41: 31$ & $-71: 36: 00$ & 210 & 37 & 0.7 & II \\
LMC11 & $05: 41: 56$ & $-70: 11: 07$ & 263 & 55 & 1.5 & III \\
LMC12 & $05: 52: 39$ & $-68: 57: 51$ & 180 & 56 & 1.5 & III \\
\hline SMC PILOT & $00: 52: 00$ & $-72: 48: 00$ & 268 & 32 & 1.5 & I \\
SMC1 & $00: 33: 45$ & $-73: 25: 07$ & $\ldots$ & $\ldots$ & $\ldots$ & $\ldots$ \\
SMC2 & $00: 49: 10$ & $-73: 51: 48$ & 273 & 53 & 1.5 & III \\
SMC3 & $00: 55: 09$ & $-72: 15: 13$ & $\ldots$ & $\ldots$ & $\ldots$ & $\ldots$ \\
SMC4 & $01: 14: 18$ & $-72: 00: 43$ & 256 & 67 & 1.5 & III \\
SMC5 & $01: 15: 12$ & $-73: 33: 57$ & 239 & 76 & 1.5 & III \\
\hline
\end{tabular}

Notes. Each field has a $1^{\circ}$ radius. $N_{\mathrm{QSO}}$ does not have to add up to 713 new QSOs and 45 known QSOs because the fields overlap slightly (see Figure 2) and a quasar can be observed in several fields.

of these fields (LMC4a as field LMC4b) during the final observing run.

We used the 580V (blue channel) and 385R (red channel) gratings to provide a resolution of $R \approx 1300$ and a spectral range of $3700-8800 \AA$, with the spectra spliced near $5700 \AA$. This broad coverage, low resolution mode is well suited for AGN identification since we are interested in relatively broad but sparse lines. The data were reduced and calibrated with the standard AAOmega 2DFDR routines (Taylor et al. 1996). We then inspected all the spectra using our own dedicated code for finding AGNs. We searched for the common redshifted AGN spectral lines (see, e.g., Vanden Berk et al. 2001) such as hydrogen Ly $\alpha$ at $1216 \AA, \mathrm{H} \delta$ at $4101 \AA, \mathrm{H} \gamma$ at $4340 \AA, \mathrm{H} \beta$ at $4861 \AA, \mathrm{H} \alpha$ at $6563 \AA$, magnesium Mg II at $2800 \AA$, carbon CIV at $1549 \AA$, and C III] at $1909 \AA$, and also the narrow lines of oxygen [O II] at $3727 \AA$ and [O III] at $4959 \AA$ and $5007 \AA$. The AGN identification was viewed as confirmed if at least two AGN lines were identified, with the exception of the redshift range from $0.7<z<1.2$ where $\mathrm{Mg}$ II is frequently the only observable line. We paid special attention to $z \approx 1$ AGNs, for which incorrect splicing of the blue and red spectra at $5700 \AA$ can mimic the $\mathrm{Mg}$ II line.

\section{NEW QUASARS}

We identified a total of 758 quasars, 565 in the LMC and 193 in the SMC, from our targeted sample of 2248 LMC and 766 SMC sources, including those reported earlier in Papers I and II. We chose targets independent of any prior identifications. Of the 66 known AGNs in our observed fields, we selected 48 as candidates, observed 46, and re-confirmed 45 (we discuss the exception below), leaving a total of 527 and 186 new MQS quasars behind the LMC and SMC, respectively. Of the 18 known AGNs that were not selected as candidates, five were in the pilot field of Paper I that was governed by a different set of selection criteria (although not observed, three of them were 

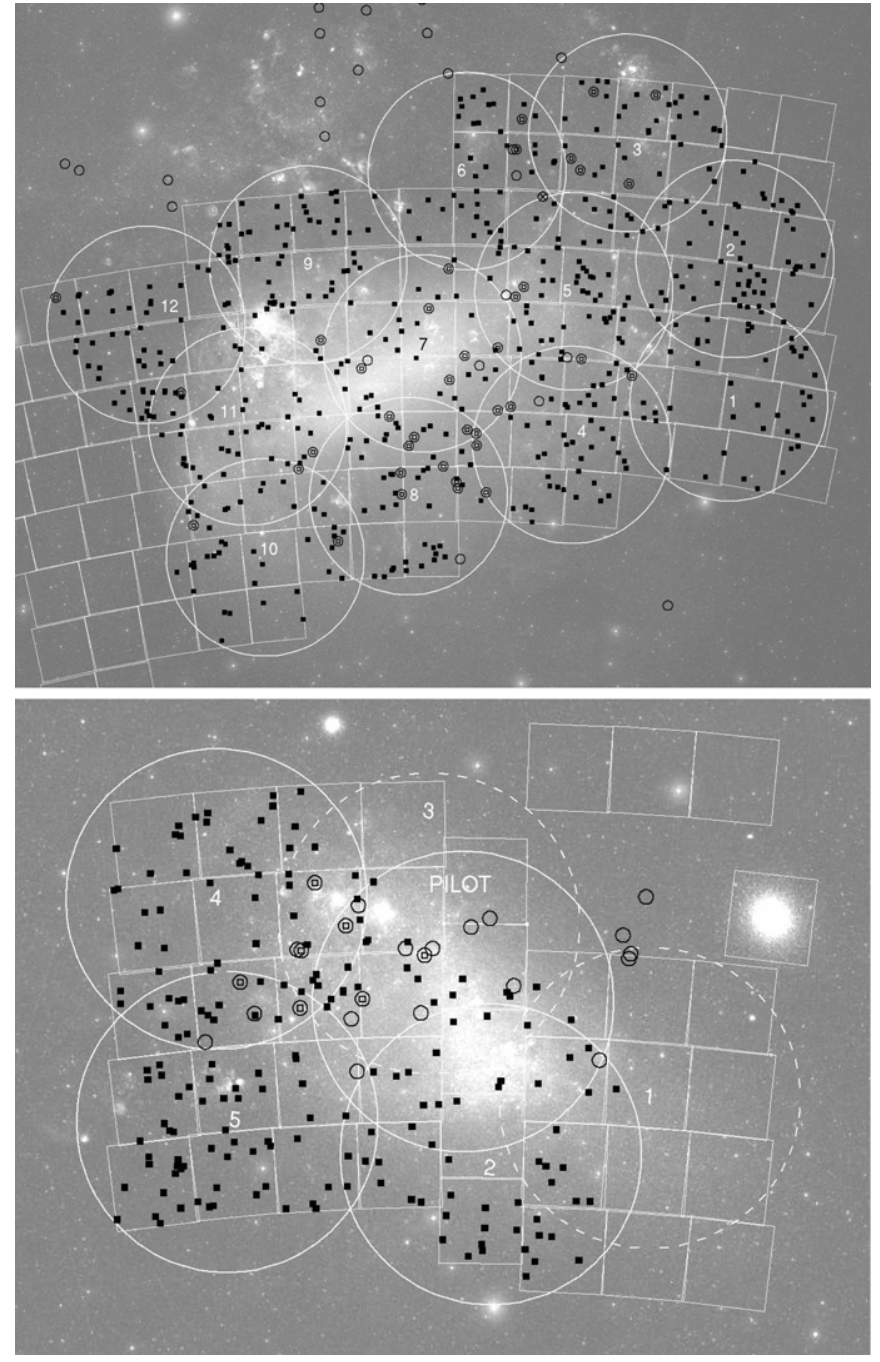

Figure 2. Twelve MQS LMC fields (top panel) and six SMC fields (bottom panel) identified by their field numbers. In addition to the three official SMC fields that were observed (solid lines; fields 2, 4, and 5), we show our pilot field and the two unobserved SMC fields as dashed circles. Black squares mark our new MQS confirmed AGNs, open circles mark previously known quasars; these objects then contain a central open square if they were reobserved and confirmed. The $\otimes$ symbol (near the overlap of the LMC 3, 5, and 6 fields) marks the one exception, the AGN from Dobrzycki et al. (2005) that we were unable to confirm. The white squares outline the OGLE-III fields. The top (bottom) image covers approximately $9^{\circ} \times 7^{\circ}\left(5^{\circ} \times 4^{\circ}\right)$. North is up and east is to the left.

included in the current candidate list; the remaining two had incomplete mid-IR colors, no X-ray emission, and insignificant optical variability). Of the 13 known AGNs in the observed standard fields, six lacked (complete) Spitzer photometry, six were not detected by Spitzer, and one lay outside the mid-IR selection region. Most (nine) were not significantly variable, although we did lose one known AGN for being "too variable" and having a structure function amplitude $A>0.4$ mag. Three AGNs were variable but fainter than $I>19.5 \mathrm{mag}$. All but three had no X-ray counterparts, and these three remaining sources had ROSAT detection probabilities below the threshold we used for our target selection.

The location of new (and previously known) AGNs on the sky is shown in Figure 2, while their basic properties and coordinates are reported in Tables 2 (LMC) and 3 (SMC). The selected spectra of 50 new MQS AGNs are shown in Figure 3. All identified AGNs had their spectra classified into quality classes: Q1 for obvious AGN spectra, Q2 for relatively obvious AGN spectra with problems/contamination, and Q3 for just above a borderline, usually low $\mathrm{S} / \mathrm{N}$ or highly contaminated AGN spectra. There are $372(282,90)$ Q1 AGNs behind the MCs (LMC, SMC), $299(217,82)$ Q2, and $87(66,21)$ Q3. For sources brighter than $I<19.5 \mathrm{mag}, 58 \%$ of them are Q1 AGNs, while for sources fainter than this limit only $35 \%$ are Q1, simply reflecting decreasing spectra quality with decreasing $\mathrm{S} / \mathrm{N}$.

One AGN from Paper II, AGN MQS J051509.61-701711.7, turned out to be a false positive, where we misidentified the rest-frame [O I] lines at $6300 \AA$ and $6364 \AA$ as the [O III] $4959 \AA$ and $5007 \AA$ lines at a redshift of $z \approx 0.27$; we therefore deleted this object from the final sample. We inspected all other AGNs at similar redshifts and found no other misidentifications. We were unable to confirm the AGN J050550.35-675017.5 from Dobrzycki et al. (2005). These authors selected this object as an X-ray source from higher resolution (than ROSAT) $X M M$-Newton data, while we selected it as a QSO-Aa mid-IR candidate. In the OGLE-III images, it is associated with a $\sim 13^{\prime \prime} \times 3^{\prime \prime}$, mildly edge-on galaxy that may have a bright nucleus. As such, the source is almost certainly an AGN. However, Dobrzycki et al. (2005) assign a redshift of a $z=0.07$ quasar based on a single noisy line interpreted as $\mathrm{H} \alpha$, and we are unable to confirm this redshift or identify an alternative. We count this source as unconfirmed in our statistical discussions. This source is marked with a " $\otimes$ " symbol in Figure 2. There are no high resolution images available from the Hubble Space Telescope (HST) archives, so it was not used in any of the HST proper motion studies.

Figures 4-6 summarize various properties of the sample. Figure 4 shows the distribution of observed and expected optical colors as a function of redshift, where we compute the expected colors and $K$-corrections using the template AGN spectrum from Vanden Berk et al. (2001). We use Galactic/MC extinction corrections from Haschke et al. (2011). We combine the $K$-corrections and the extinction corrections to estimate the absolute magnitude of each AGN assuming a standard $\Lambda$ CDM cosmological model with $\left(\Omega_{\Lambda}, \Omega_{M}, \Omega_{k}\right)=(0.7,0.3,0.0)$ and $h=H_{0} /\left(100 \mathrm{~km} \mathrm{~s}^{-1} \mathrm{Mpc}^{-1}\right)=0.71$ to calculate luminosity distances. Figure 5 shows the distribution of the AGNs in absolute $V$-band (top panel) and $I$-band (middle panel) magnitudes along with a histogram of the overall redshift distribution (bottom panel). Finally, Figure 6 shows the cumulative surface density of the sample as a function of $I$-band magnitude. If we compare this result to the SDSS $i$-band number counts from Richards et al. (2006), corrected to the OGLE I-band (shifted by $-0.3 \mathrm{mag}$ ), we see that the MQS sample is roughly $\sim 75 \%$ complete for $I<19 \mathrm{mag}$, which seems quite good given the nature of the survey fields! Some of the incompleteness is associated with regions of very high stellar density, as illustrated by the lower number of quasars directly behind the central regions of the MCs.

\section{UNIDENTIFIED AND CONTAMINATING SOURCES}

The remaining LMC (SMC) sources for which we obtained spectra can be divided into 1017 (344) contaminating sources and 667 (229) objects with featureless spectra, where a contaminating source is clearly some sort of stellar source in the LMC and a featureless spectrum is one where the $\mathrm{S} / \mathrm{N}$ is simply too poor to propose a classification. In Paper II, we investigated the nature of the contaminating sources and found that they are typically planetary nebulae (PNe), YSOs, B/Be stars, etc., as might be expected from the requirement that they show dust 
Table 2

MQS Quasars Behind the LMC

\begin{tabular}{|c|c|c|c|c|c|c|c|c|c|c|c|c|c|}
\hline MQS AGN Name & $\begin{array}{c}\text { R.A. } \\
\text { (deg) } \\
(2)\end{array}$ & \multicolumn{2}{|c|}{$\begin{array}{c}\text { Decl. } \\
(\mathrm{deg}) \\
(3)\end{array}$} & (4) & $\begin{array}{c}\mu \\
(\mathrm{mag}) \\
(5)\end{array}$ & $\begin{array}{c}V \\
(\mathrm{mag}) \\
(6)\end{array}$ & $\begin{array}{c}I \\
(\mathrm{mag}) \\
(7)\end{array}$ & $\begin{array}{c}A_{V} \\
(\mathrm{mag}) \\
(8)\end{array}$ & $\begin{array}{c}A_{I} \\
(\mathrm{mag}) \\
(9)\end{array}$ & $\begin{array}{c}K_{V} \\
(\mathrm{mag}) \\
(10)\end{array}$ & $\begin{array}{c}K_{I} \\
(\mathrm{mag}) \\
(11)\end{array}$ & $\begin{array}{c}M_{V} \\
(\mathrm{mag}) \\
(12)\end{array}$ & $\begin{array}{c}M_{I} \\
(\mathrm{mag}) \\
(13)\end{array}$ \\
\hline MQS J043110.08-695241.5 & 67.792000 & \multicolumn{2}{|c|}{-69.878194} & 1.548 & 45.19 & 19.63 & 18.89 & 0.29 & 0.17 & -0.40 & -0.07 & -25.45 & -26.40 \\
\hline MQS J043151.34-692437.9 & 67.963917 & \multicolumn{2}{|c|}{-69.410528} & 0.594 & 42.60 & 19.77 & 19.01 & 0.22 & 0.13 & -0.21 & 0.18 & -22.84 & -23.90 \\
\hline MQS J043200.60-693846.5 & 68.002500 & \multicolumn{2}{|c|}{-69.646250} & 1.409 & 44.93 & 20.74 & 19.89 & 0.22 & 0.13 & -0.38 & -0.01 & -24.03 & -25.17 \\
\hline MQS J043221.19-701129.5 & 68.088292 & \multicolumn{2}{|c|}{-70.191528} & 0.957 & 43.90 & 17.62 & 17.26 & 0.29 & 0.17 & -0.42 & 0.23 & -26.15 & -27.04 \\
\hline MQS J043232.77-694433.2 & 68.136542 & \multicolumn{2}{|c|}{-69.742556} & 2.162 & 46.07 & 19.01 & 18.15 & 0.22 & 0.13 & -0.59 & -0.19 & -26.69 & -27.87 \\
\hline MQS J043238.16-700438.4 & 68.159000 & \multicolumn{2}{|c|}{-70.077333} & 2.145 & 46.06 & 19.73 & 18.91 & 0.22 & 0.13 & -0.59 & -0.19 & -25.96 & -27.09 \\
\hline MQS J043259.64-693653.0 & 68.248500 & \multicolumn{2}{|c|}{-69.614722} & 0.948 & 43.87 & 19.79 & 19.28 & 0.22 & 0.13 & -0.42 & 0.23 & -23.89 & -24.95 \\
\hline MQS J043308.66-701341.5 & 68.286083 & \multicolumn{2}{|c|}{-70.228194} & 1.428 & 44.97 & 17.91 & 17.13 & 0.29 & 0.17 & -0.38 & -0.01 & -26.97 & -28.00 \\
\hline MQS J043322.97-680832.9 & 68.345708 & \multicolumn{2}{|c|}{-68.142472} & 0.937 & 43.84 & 20.89 & 20.66 & 0.12 & 0.07 & -0.41 & 0.23 & -22.66 & -23.49 \\
\hline MQS J043330.96-690844.0 & 68.379000 & \multicolumn{2}{|c|}{-69.145556} & 3.028 & 46.96 & 19.28 & 18.72 & 0.24 & 0.14 & -0.77 & -0.27 & -27.16 & -28.11 \\
\hline MQS AGN Name & \multicolumn{2}{|c|}{$\begin{array}{l}\text { OGLE-III } \\
\text { ID } \\
(14)\end{array}$} & \multicolumn{2}{|c|}{$\begin{array}{c}\text { KK09 } \\
\text { Class } \\
(15)\end{array}$} & Mid-IR & \multicolumn{2}{|c|}{ X-Ray } & Var. & Notes & $\begin{array}{c}\text { Quality } \\
\text { Flag } \\
(20)\end{array}$ & & Emission & ines \\
\hline MQS J043110.08-695241.5 & $1 \mathrm{mc} 157$. & 2453 & QS & $-\mathrm{Aa}$ & 1 & & & 1 & $\mathrm{~N}$ & Q2 & & $\mathrm{C}$ III], $\mathrm{I}$ & \\
\hline MQS J043151.34-692437.9 & $\operatorname{lmc} 156$. & 1036 & QS & $-\mathrm{Aa}$ & 1 & & & 1 & $\mathrm{~N}$ & Q1 & & Mg II, [O II], & $\beta,[\mathrm{O} \mathrm{III}]$ \\
\hline MQS J043200.60-693846.5 & $\operatorname{lmc} 157$. & 3272 & QS & -Aa & 1 & & & 1 & $\mathrm{~N}$ & Q2 & & $\mathrm{Mg}$ & \\
\hline MQS J043221.19-701129.5 & $\operatorname{lmc} 158$ & 202 & YS & -Aa & 1 & & & 1 & $\mathrm{~N}$ & Q3 & & $\mathrm{Mg}$ & \\
\hline MQS J043232.77-694433.2 & $\operatorname{lmc} 157$ & 244 & QS & -Aa & 1 & & & 1 & $\mathrm{~N}$ & Q1 & & Ly $\alpha$, Si IV, C & $\mathrm{V}, \mathrm{C}$ III] \\
\hline MQS J043238.16-700438.4 & $\operatorname{lmc} 157$ & 247 & QS & -Aa & 1 & & & 1 & $\mathrm{~N}$ & Q1 & & Ly $\alpha$, Si IV, C & $\mathrm{V}, \mathrm{C}$ III] \\
\hline MQS J043259.64-693653.0 & $\operatorname{lmc} 157$ & 758 & QS & -Aa & 1 & & & 1 & $\mathrm{~N}$ & Q2 & & Mg II, [ & \\
\hline MQS J043308.66-701341.5 & $\operatorname{lmc} 158$. & 2814 & YS & -Aa & 1 & & & 1 & $\mathrm{~N}$ & Q1 & & $\mathrm{C}$ III], $\mathrm{I}$ & \\
\hline MQS J043322.97-680832.9 & $\operatorname{lmc} 154$. & 692 & QS & -Aa & 1 & & & 0 & $\mathrm{~N}$ & Q3 & & $\mathrm{Mg}$ & \\
\hline MQS J043330.96-690844.0 & $1 \mathrm{mc} 156$. & 143 & QS & -Aa & 1 & & & 1 & $\mathrm{~N}$ & Q3 & & $\operatorname{Ly} \alpha$, Si I & C IV \\
\hline
\end{tabular}

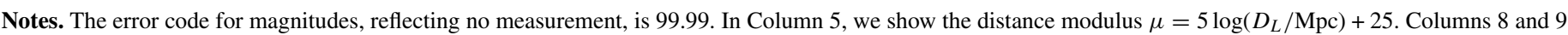

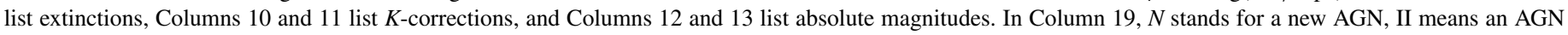
reported in Paper II, and $K$ is for an already known AGN.

(This table is available in its entirety in a machine-readable form in the online journal. A portion is shown here for guidance regarding its form and content.)

emission, variability, or X-ray emission. The properties of the final larger sample will be explored elsewhere. Figure 6 compares the cumulative distributions of these sources to those of the AGNs. We see that contaminating sources dominate the overall target distribution at bright magnitudes and that featureless sources dominate at faint magnitudes. Essentially, filling the fibers means we can look at all possible bright candidates and gamble that we might identify quasars fainter than $I \sim 20$ mag despite the high effective sky backgrounds. This technique leads to a low apparent detection efficiency of $\sim 30 \%$, but is really just a consequence of using all the available fibers.

\section{AGN SELECTION METHODS}

We can use the overall sample to explore the various search methods proposed to identify quasars behind the MCs. In some sense, this question is almost unnecessary, since the MQS has already identified the majority of bright quasars behind the densest regions of the MCs and the problem becomes simpler in any expansion of the search region because the stellar densities are lower. These issues would be relevant, however, to attempts to find fainter quasars, although there is no immediately obvious scientific driver for such a search. Table 4 summarizes the statistics for the various methods, where readers should focus on the differences in the efficiencies rather than the absolute efficiencies since the latter are by definition low because of our strategy of using every fiber. Note that the discussion of efficiency and statistics uses only the results from the observed fields and excludes the unobserved SMC fields and the SMC test field.
For the present analysis, we will discuss relative completenesses more carefully than in Paper II. The extra complication is that we assigned quasars an observational priority based on whether they were selected based on $i=1,2$, or 3 methods, so the fraction observed $f_{i}$ depends on $i$. While there were three ways a candidate selected by only $i=1$ (mid-IR, variability, or X-ray) or $i=2$ (mid-IR/variability, mid-IR/X-ray, or variability/X-ray) methods would be included in the sample, the probability of assigning a fiber did not depend on which of the cases applied. As a result, for $i=1$ and $i=2$ we need only one correction factor and not a separate correction for each of the possible sub-cases. For any particular class of objects (e.g., QSO-Aa), there were then $N_{i}$ candidates yielding $Q_{i}$ quasars, so the overall efficiency for the class is

$$
E=\left[\sum_{i} Q_{i} f_{i}^{-1}\right]\left[\sum_{i} N_{i}\right]^{-1}
$$

Note that the total number of candidates is $N_{i}=O_{i} / f_{i}$, where $O_{i}$ is the number of candidates that were observed. Therefore, if all priorities were observed with equal probability $\left(f_{1}=f_{2}=f_{3}\right)$, the efficiency is simply the number of quasars found divided by the number of objects observed. For example, if we consider all 3106 mid-IR candidates in the observed fields, there were 2173 , 829 , and 104 selected by the $i=1,2$, or 3 methods, of which we observed 1703,754 , and 98 , corresponding to $f_{1}=0.78$, $f_{2}=0.91$, and $f_{3}=0.94$.

Figure 1 shows the mid-IR selection criteria we introduced in Kozłowski \& Kochanek (2009). Table 4 summarizes the various 


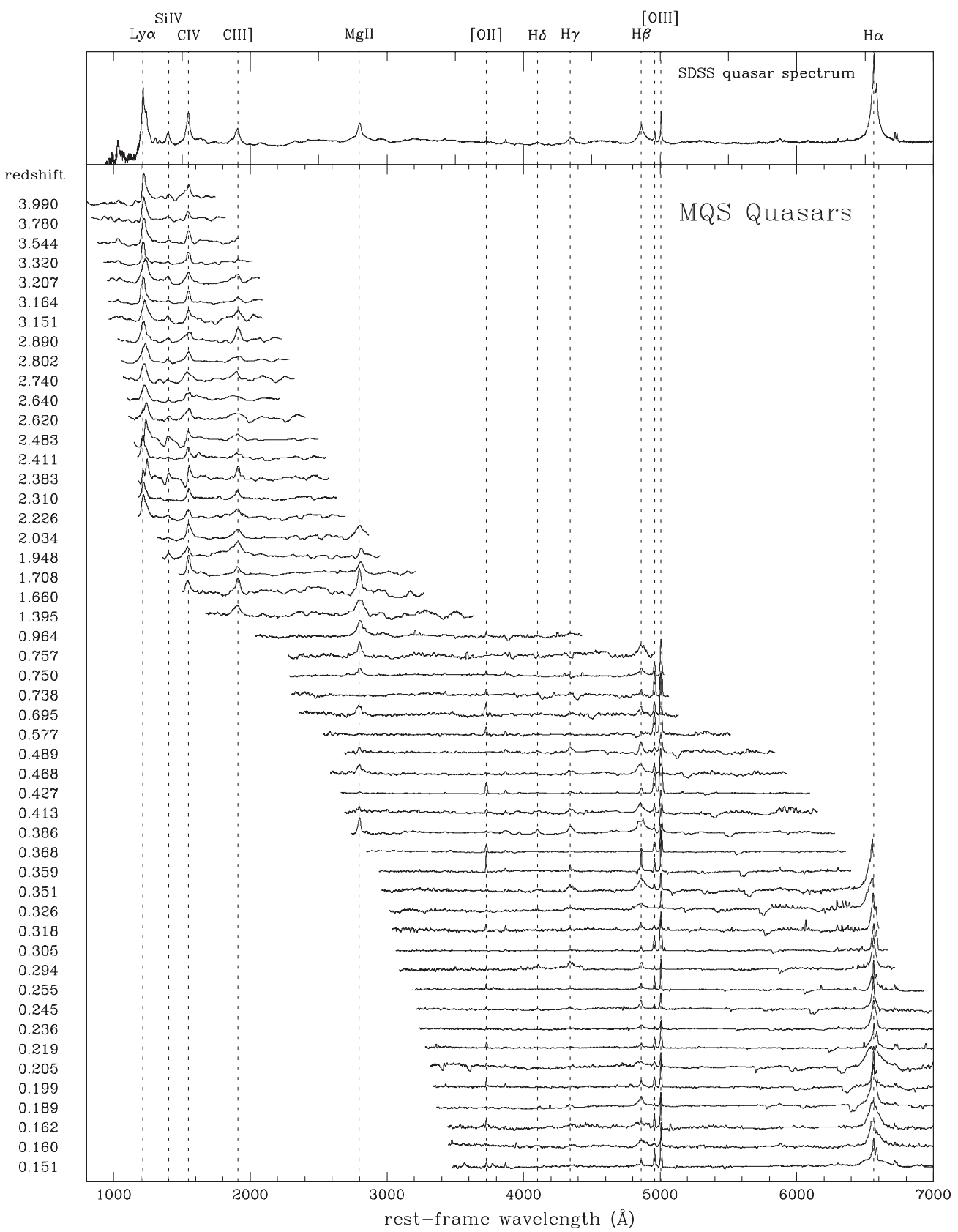

Figure 3. Main panel: rest-frame spectra of 50 out of the 713 new LMC/SMC AGNs reported in this paper. Except for the Quality 2 spectra used for the examples at $z=2.640,2.740$, and 3.151, these are all Quality 1 spectra. These spectra have been flattened, smoothed, and scaled. The majority of the $z \approx 0$ LMC/SMC emission lines as well as some of the atmospheric absorption features have been masked in order to emphasize the quasar emission lines. Each spectrum is labeled by redshift (on the left) and we also mark the common quasar lines (vertical dashed lines with labels). Top panel: for comparison, we show the composite quasar spectrum (detrended, flattened, and scaled) based on 2200 spectra from SDSS (Vanden Berk et al. 2001).

mid-IR selection groups, where in our discussion we will ignore those with few observed sources (e.g., YSO-Ba). As expected, the highest yield is for QSO-Aa objects ( $29 \%)$, followed by QSO-Ba $(\sim 24 \%)$. These results are driven by our inclusion of faint sources; if we restrict the sample to $I<19.5 \mathrm{mag}$ (the bright sample, hereafter), the efficiency rises to $49 \%$ and $51 \%$, respectively. As expected, the YSO regions have lower yields $(\sim 20 \%)$ and the yields become very low $(<10 \%)$ if a target did not have the typical optical/mid-IR color of quasars in the AGES (Kochanek et al. 2012) survey (class "b" rather than class "a"). It appears that the distinction between sources along the blackbody color track (class B) as compared to those off that color track (class A) has little effect and could simply be dropped. Overall, the yield for a source satisfying any of the mid-IR criteria was $27 \%$ for all sources and $44 \%$ for bright sources ("Mid-IR (any)" in Table 4). Interestingly, if we restrict the sample to mid-IR selected candidates that were not also selected based on their variability or X-ray properties ("Mid-IR (only)"), the overall yield is still $18 \%$ (27\% bright). This result means that a large fraction of the mid-IR-selected quasars are not being selected by the variability or X-ray criteria. At least for the latter, Hickox et al. (2009) and Assef et al. (2010) have previously noted that X-ray and mid-IR selection methods tend to select different sources.

We discuss the variability selection results in three parts. First, we consider the variability selection as actually used to select 


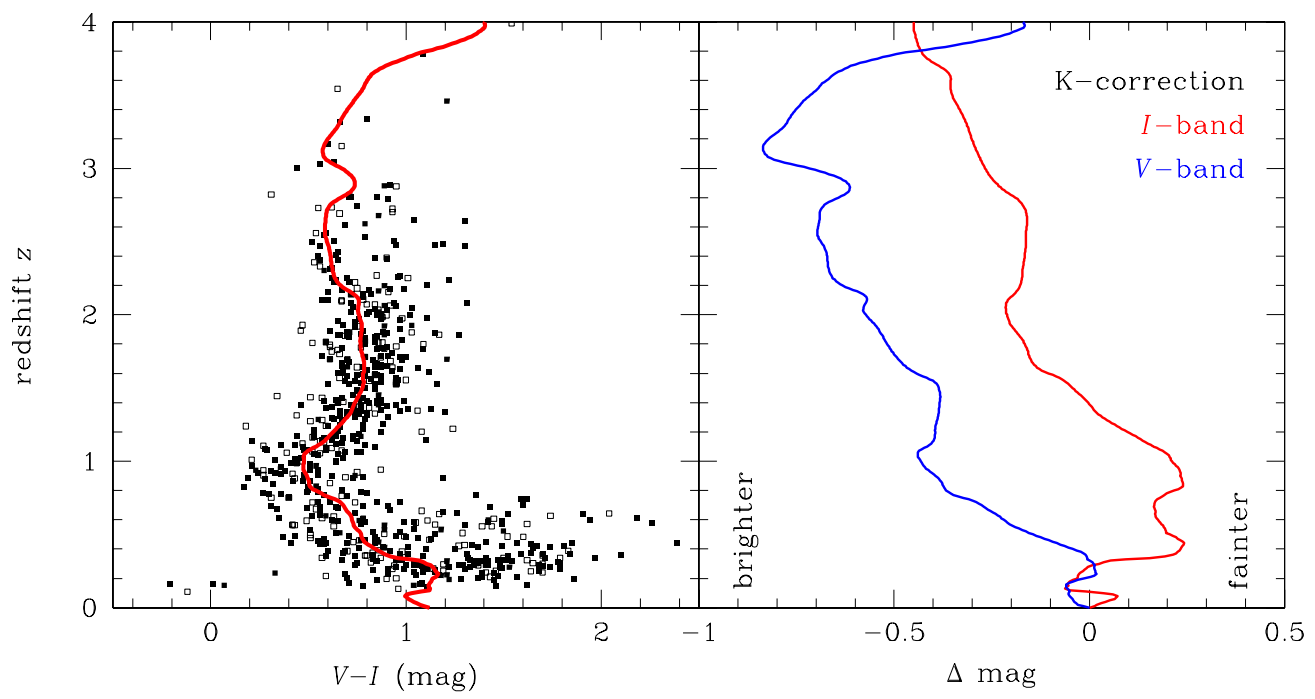

Figure 4. AGN colors (left panel) and $K$-corrections (right panel) as a function of redshift $z$. Left panel: filled (open) squares represent the LMC (SMC) quasars confirmed by the MQS. The red line is the expected $V-I$ color derived from the average SDSS quasar spectrum from Vanden Berk et al. (2001) as it is redshifted through the OGLE filters. Significant outliers from this line are AGNs blended with stellar light from the MCs. Lower luminosity AGNs at lower redshifts are frequently redder because of increased contamination from their host galaxies. Right panel: AGN $K$-corrections for the $V$-band (blue) and $I$-band (red) OGLE filters.

Table 3

MQS Quasars Behind the SMC

\begin{tabular}{|c|c|c|c|c|c|c|c|c|c|c|c|c|}
\hline $\begin{array}{l}\text { MQS AGN Name } \\
\text { (1) }\end{array}$ & $\begin{array}{l}\text { R.A. } \\
\text { (deg) } \\
(2)\end{array}$ & $\begin{array}{c}\text { Decl. } \\
(\mathrm{deg}) \\
(3)\end{array}$ & (4) & $\begin{array}{c}\mu \\
(\mathrm{mag}) \\
(5)\end{array}$ & $\begin{array}{c}V \\
(\mathrm{mag}) \\
(6)\end{array}$ & $\begin{array}{c}I \\
(\mathrm{mag}) \\
(7)\end{array}$ & $\begin{array}{c}A_{V} \\
(\mathrm{mag}) \\
(8)\end{array}$ & $\begin{array}{c}A_{I} \\
(\mathrm{mag}) \\
(9)\end{array}$ & $\begin{array}{c}K_{V} \\
(\mathrm{mag}) \\
(10)\end{array}$ & $\begin{array}{c}K_{I} \\
(\mathrm{mag}) \\
(11)\end{array}$ & $\begin{array}{c}M_{V} \\
(\mathrm{mag}) \\
(12)\end{array}$ & $\begin{array}{c}M_{I} \\
(\mathrm{mag}) \\
(13)\end{array}$ \\
\hline MQS J003704.67-732229.6 & 9.269458 & -73.374889 & 0.750 & 43.24 & 18.89 & 18.49 & 0.05 & 0.03 & -0.32 & 0.18 & -24.08 & -24.96 \\
\hline MQS J003857.54-741000.9 & 9.739750 & -74.166917 & 2.692 & 46.65 & 18.41 & 17.75 & 0.12 & 0.07 & -0.69 & -0.16 & -27.67 & -28.81 \\
\hline MQS J003942.32-732428.1 & 9.926333 & -73.407806 & 0.382 & 41.46 & 20.08 & 19.10 & 0.07 & 0.04 & -0.02 & 0.23 & -21.43 & -22.63 \\
\hline MQS J003947.82-743444.8 & 9.949250 & -74.579111 & 1.810 & 45.60 & 18.43 & 17.55 & 0.12 & 0.07 & -0.52 & -0.17 & -26.77 & -27.95 \\
\hline MQS J003957.65-730603.6 & 9.990208 & -73.101000 & 0.569 & 42.51 & 19.85 & 19.43 & 0.10 & 0.06 & -0.20 & 0.18 & -22.56 & -23.33 \\
\hline MQS J004023.71-741013.9 & 10.098792 & -74.170528 & 0.623 & 42.74 & 19.29 & 18.66 & 0.12 & 0.07 & -0.23 & 0.17 & -23.34 & -24.32 \\
\hline MQS J004143.75-731017.1 & 10.432292 & -73.171417 & 0.217 & 40.10 & 21.55 & 20.96 & 0.12 & 0.07 & 0.01 & -0.03 & -18.68 & -19.18 \\
\hline MQS J004145.04-725435.9 & 10.437667 & -72.909972 & 0.267 & 40.60 & 20.19 & 19.05 & 0.12 & 0.07 & 0.01 & -0.01 & -20.55 & -21.62 \\
\hline MQS J004152.35-735626.8 & 10.468125 & -73.940778 & 0.422 & 41.72 & 21.57 & 20.30 & 0.10 & 0.06 & -0.06 & 0.24 & -20.19 & -21.72 \\
\hline MQS J004241.66-734041.3 & 10.673583 & -73.678139 & 0.905 & 43.76 & 20.18 & 19.91 & 0.10 & 0.06 & -0.40 & 0.23 & -23.28 & -24.14 \\
\hline MQS AGN Name & \multicolumn{2}{|c|}{$\begin{array}{l}\text { OGLE-III } \\
\text { ID } \\
(14)\end{array}$} & $\begin{array}{c}\text { KK09 } \\
\text { Class } \\
(15) \\
\end{array}$ & Mid-IR & & K-Ray & $(18)$ & Notes & \multicolumn{2}{|c|}{$\begin{array}{l}\text { Quality } \\
\text { Flag } \\
(20)\end{array}$} & \multicolumn{2}{|c|}{ Emission Lines } \\
\hline MQS J003704.67-732229.6 & \multicolumn{2}{|c|}{ smc130.2.11076 } & QSO-Aa & 1 & & 0 & 1 & $\mathrm{~N}$ & \multicolumn{2}{|c|}{ Q2 } & \multicolumn{2}{|c|}{ Mg II } \\
\hline MQS J003857.54-741000.9 & \multicolumn{2}{|c|}{ smc128.8.594 } & QSO-Aa & 1 & & 0 & 1 & $\mathrm{~N}$ & \multicolumn{2}{|c|}{$\mathrm{Q} 1$} & \multicolumn{2}{|c|}{ Ly $\alpha$, Si IV, C IV, C III] } \\
\hline MQS J003942.32-732428.1 & \multicolumn{2}{|c|}{ smc125.7.5747 } & QSO-Aa & 1 & & 0 & 1 & $\mathrm{~N}$ & \multicolumn{2}{|c|}{ Q1 } & \multicolumn{2}{|c|}{$[\mathrm{OII}], \mathrm{H} \beta,[\mathrm{O} \mathrm{III}]$} \\
\hline MQS J003947.82-743444.8 & \multicolumn{2}{|c|}{ smc129.7.2762 } & QSO-Aa & 1 & & 0 & 0 & $\mathrm{~N}$ & \multicolumn{2}{|c|}{ Q1 } & \multicolumn{2}{|c|}{$\left.\mathrm{C}_{\text {IV }}, \mathrm{C}_{\mathrm{III}}\right], \mathrm{Mg}_{\mathrm{II}}$} \\
\hline MQS J003957.65-730603.6 & \multicolumn{2}{|c|}{ smc125.5.6063 } & QSO-Aa & 1 & & 1 & 1 & I & \multicolumn{2}{|c|}{ Q2 } & \multicolumn{2}{|c|}{$\mathrm{Mg}$ II, [O III] } \\
\hline MQS J004023.71-741013.9 & \multicolumn{2}{|c|}{$\mathrm{smc} 128.8 .9401$} & QSO-Aa & 1 & & 0 & 0 & $\mathrm{~N}$ & \multicolumn{2}{|c|}{ Q1 } & \multicolumn{2}{|c|}{$\mathrm{Mg}$ II } \\
\hline MQS J004143.75-731017.1 & \multicolumn{2}{|c|}{ smc125.5.18504 } & QSO-Aa & 1 & & 0 & 0 & $\mathrm{~N}$ & \multicolumn{2}{|c|}{ Q2 } & \multicolumn{2}{|c|}{$[\mathrm{O} \mathrm{II}],[\mathrm{O} \mathrm{III}]$} \\
\hline MQS J004145.04-725435.9 & \multicolumn{2}{|c|}{ smc126.8.16111 } & QSO-Aa & 1 & & 1 & 0 & I & \multicolumn{2}{|c|}{ Q1 } & \multicolumn{2}{|c|}{$[\mathrm{OII}], \mathrm{H} \beta,[\mathrm{O} \mathrm{III}]$} \\
\hline MQS J004152.35-735626.8 & \multirow{2}{*}{\multicolumn{2}{|c|}{ smc128.2.2551 }} & QSO-Aa & 1 & & 0 & 0 & $\mathrm{~N}$ & \multicolumn{2}{|c|}{ Q3 } & \multicolumn{2}{|c|}{ [O II], [O III] } \\
\hline MQS J004241.66-734041.3 & & & QSO-Aa & 1 & & 1 & 0 & $\mathrm{~N}$ & & & $\mathrm{Mg}$ & \\
\hline
\end{tabular}

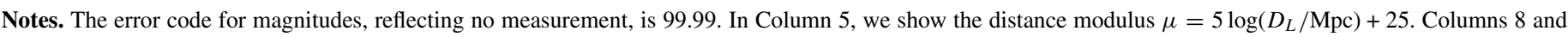

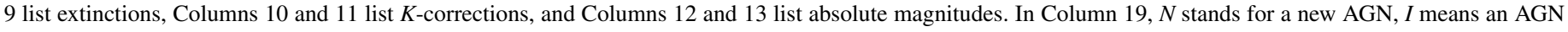
reported in Paper I, and $K$ is for an already known AGN.

(This table is available in its entirety in a machine-readable form in the online journal. A portion is shown here for guidance regarding its form and content.)

candidates and then we discuss the consequences of adding the tighter restrictions of either Kozłowski et al. (2010) or Schmidt et al. (2010). Figure 7 shows four examples of the OGLE-III light curves of newly identified quasars.

For the variability selection as implemented, we started with 50 million MC sources. After applying Cuts 1 and 2 $\left(\ln L_{\text {best }}>\ln L_{\text {noise }}+2\right.$ and 16.0/16.5 $<I<19.5 \mathrm{mag}$ ),
680,000 possibly variable sources remained. Adding the restriction on DRW timescales $(1<\log (\tau /$ days $)<5)$ reduced this number to 260,000 sources and then only 37,000 sources remained after Cuts 4 and $5(0.1<\gamma<0.9$; $A<0.4 \mathrm{mag})$ were applied. There are still large numbers of false positives, primarily "ghost variables" where fainter stars pick up a variability signal because they lie in the 
The Astrophysical Journal, 775:92 (13pp), 2013 October 1

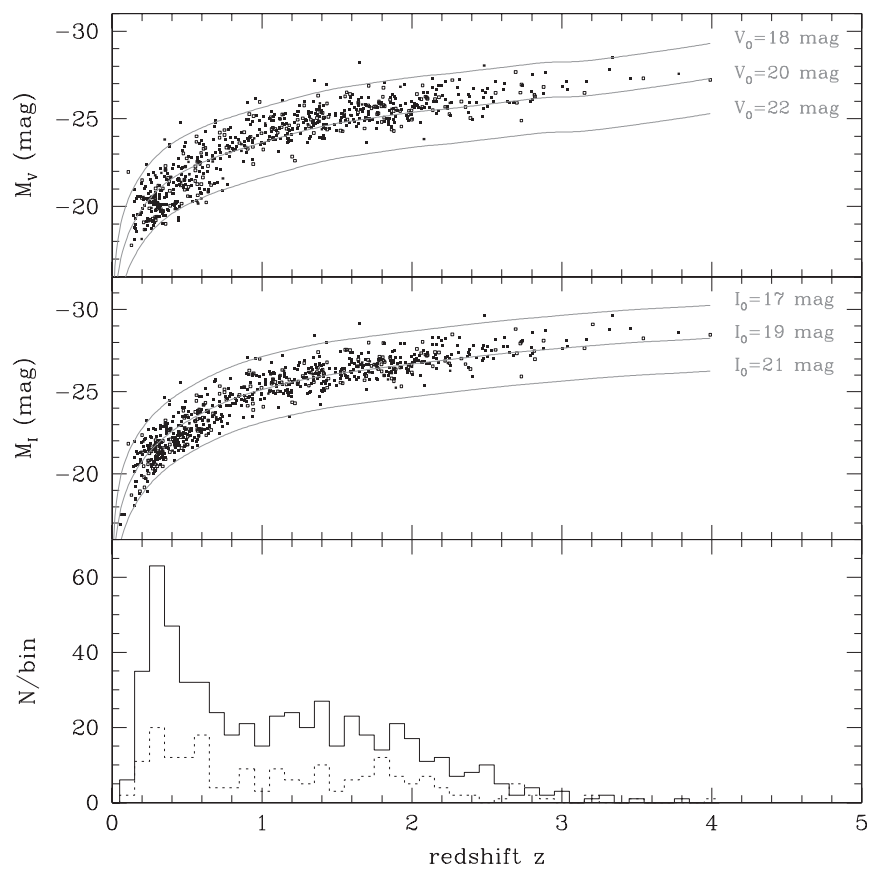

Figure 5. Absolute magnitudes of the MQS AGNs in the $V$-band (top panel) and the $I$-band (middle panel). Solid (open) squares are for the LMC (SMC) AGNs. The curves are the absolute magnitudes corresponding to constant observed magnitudes $V_{0} / I_{0}$ after adding the redshift-dependent distances and AGN $K$-corrections. The bottom panel shows redshift histograms of the confirmed quasars behind the LMC (solid line) and the SMC (dotted line).

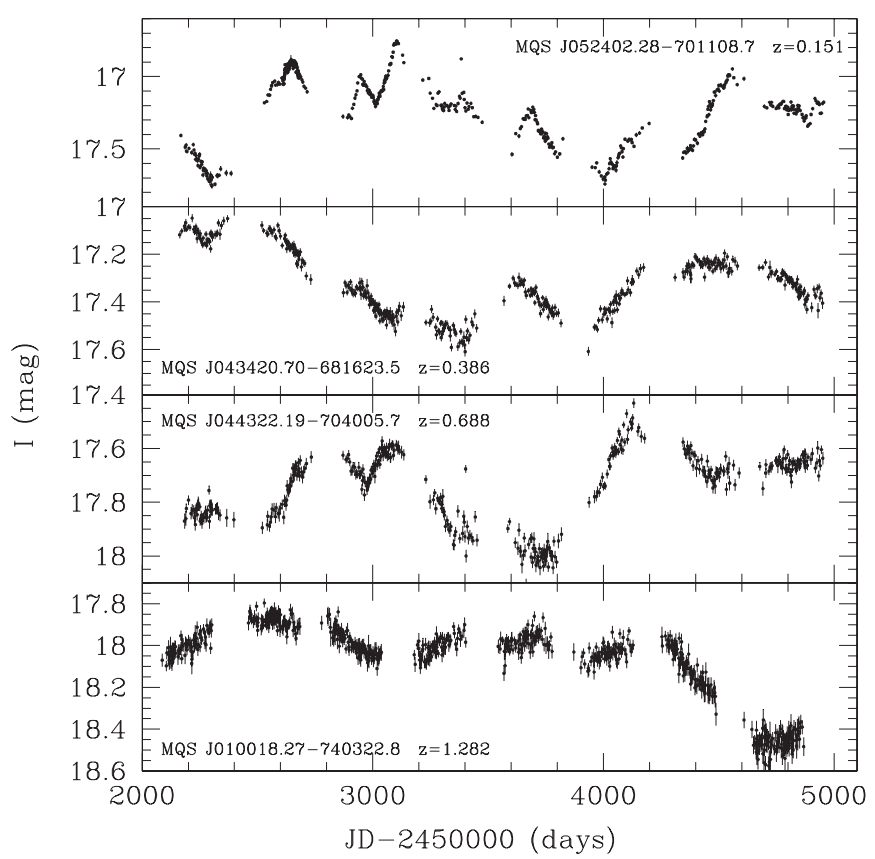

Figure 7. Four examples of OGLE-III light curves for new MQS quasars (labeled in the panels).

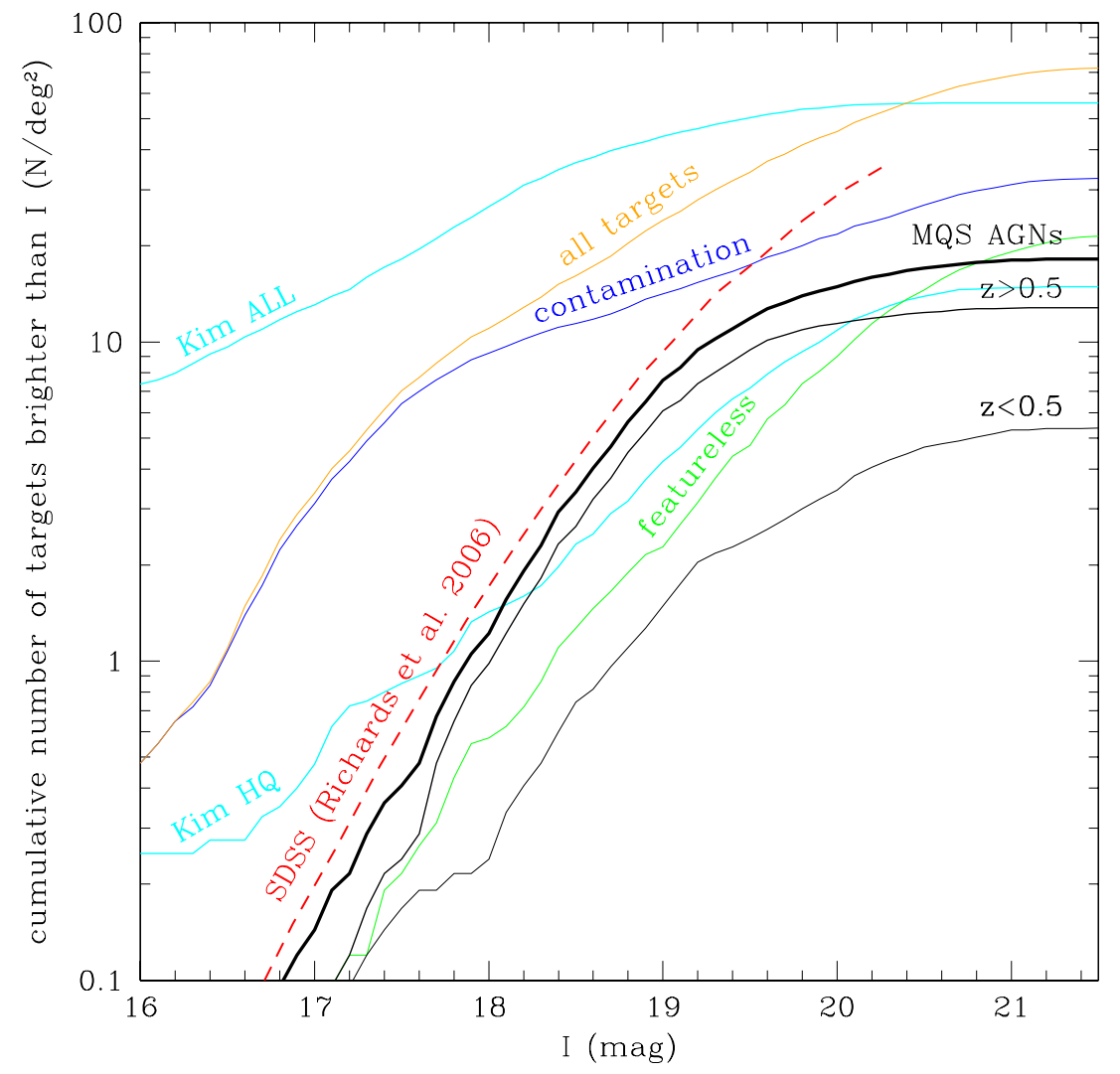

Figure 6. Cumulative distribution of the MQS AGNs above a given I-band magnitude (thick black line) compared with SDSS quasars (Richards et al. (2006), converted to $I$-band; red). The MQS survey is roughly $\sim 75 \%$ complete for $I<19$ mag. We also show the cumulative distributions of $z<0.5$ and $z>0.5$ MQS AGNs (narrow black lines), all targets (orange line), contaminating sources (blue line), and featureless sources (green line). Finally, we show the distributions of the full (Kim ALL) and "high quality" (Kim HQ) samples from Kim et al. (2012) in cyan. To put these surface densities in perspective, the density of AAOmega fibers $\left(\sim 130\right.$ fibers deg ${ }^{-2}$ ) lies above the upper scale of the figure. 


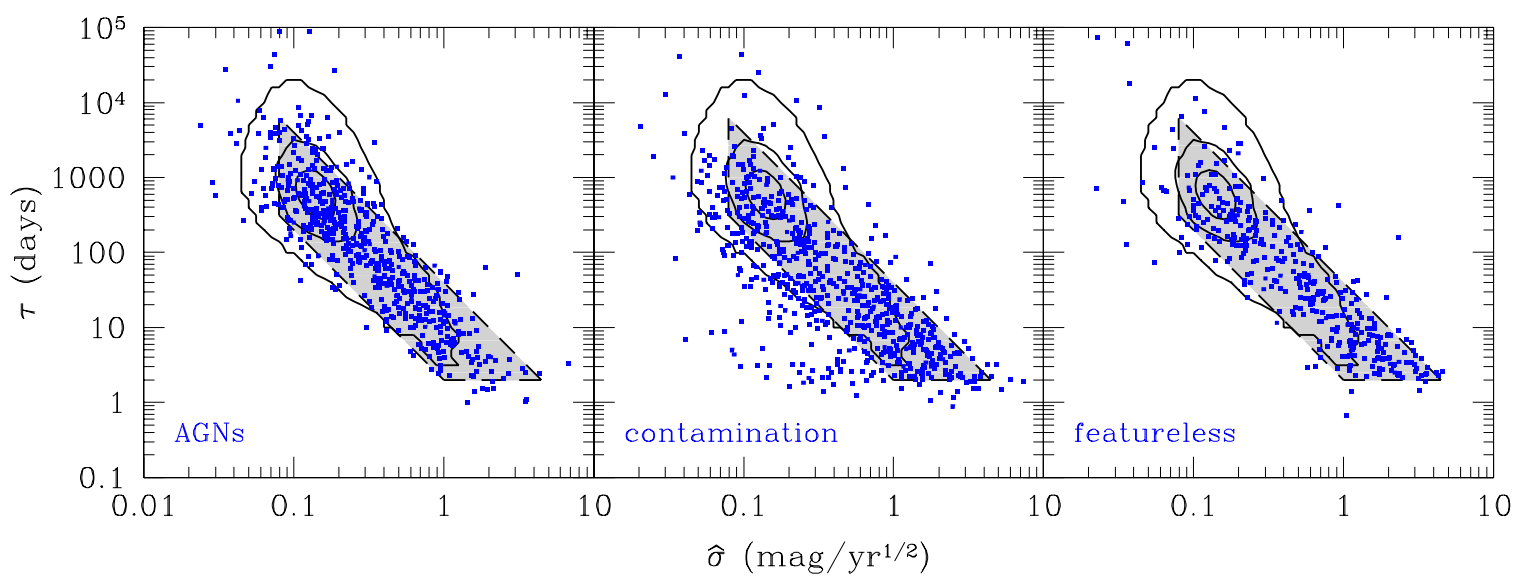

Figure 8. $\tau-\hat{\sigma}$ (timescale-modified amplitude) variability plane as defined in Kozłowski et al. (2010). In the left, middle, and right panels, we show MQS AGNs, contaminating sources, and objects with featureless spectra, respectively. We show the Kozłowski et al. (2010) trapezoid AGN selection region (gray area) and density contours $(1,10$, and 20 per 0.1 dex bins in both axes) for $\sim 9000$ variable SDSS AGNs from MacLeod et al. (2010). The Kozłowski et al. (2010) cut was designed to return high purity samples given the variability properties of contaminating stars. We extended this selection region (see Section 2 ) to probe the $\tau-\hat{\sigma}$ variability plane. The trapezoid contains $77 \%$ of the variability-selected confirmed AGNs and 59\% of all confirmed AGNs.

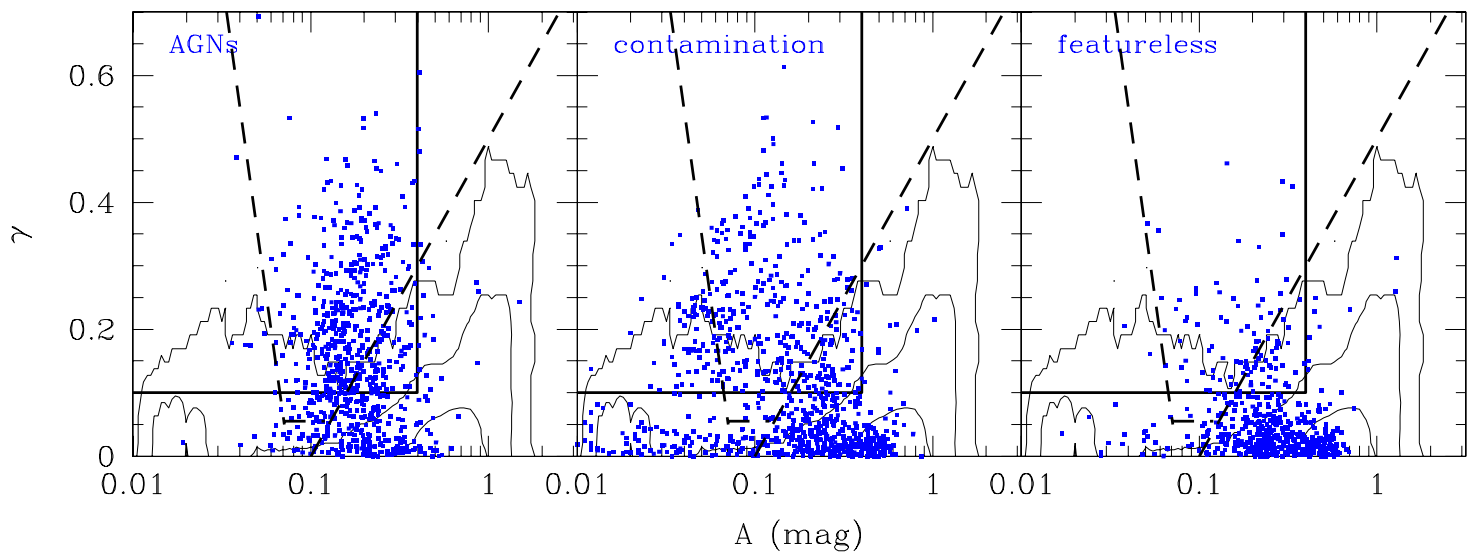

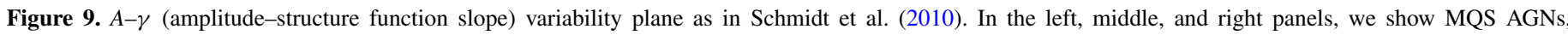

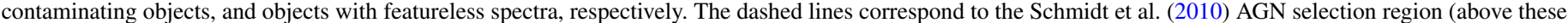

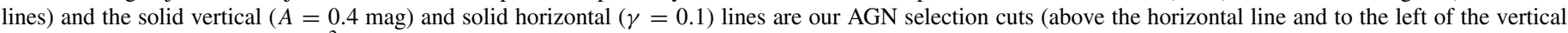

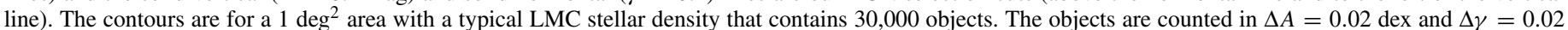

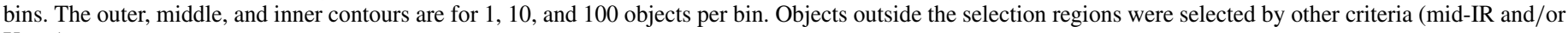
X-ray).

extended point-spread function wings of brighter variable stars. After visually inspecting this final list, we were left with the $\sim 1400$ real candidates. The resulting efficiency is quite good, with $34 \%$ of these variability-selected candidates confirmed as AGNs.

We cannot retrospectively impose the exact selection procedures we introduced in Kozłowski et al. (2010) because of the additional selection cuts we introduced in Paper II and continued to use here. We can, however, examine the effects of the additional restrictions on $\tau$ and $\sigma$ from Kozłowski et al. (2010) on the present sample, as shown in Figure 8. This figure shows the distribution of our confirmed AGNs in the space for the DRW parameters along with the selection region proposed in Kozłowski et al. (2010). A very high fraction (77\%) of all variability-selected MQS quasars (59\% of all confirmed AGNs) lie in this narrower selection region, as we would also expect given the parameter distribution of the SDSS Stripe 82 quasars from MacLeod et al. (2010). If we apply the remaining cuts from Kozłowski et al. (2010) on the variability amplitude as a function of magnitude but not the cuts on the ratio of the $V$ - and $I$-band variability amplitudes, $74 \%$ of the sample remains $(58 \%$ of all confirmed AGNs). The level of contamination seen in Figure 8 looks higher than that in Kozłowski et al. (2010) because there we only showed the distribution of other variable sources from the $\sim 2 \mathrm{deg}^{2}$ analyzed for variability by OGLE-II (Udalski et al. 1997) rather than the full OGLE-III sample (Udalski et al. 2008c). Overall, the yield for variable sources (Equation (1)) is 34\% ("Var (any)"), but in this restricted region of the $\tau-\hat{\sigma}$ plane (also using the remaining Kozłowski et al. 2010 cuts), it is $~ 45 \%$ ("Var (any) + DRW") and by definition these are all bright $16.0 / 16.5<I<19.5$ mag sources. Almost all the confirmed variability-selected AGNs were also selected as mid-IR candidates, probably because they were all also required to be relatively bright. As a result, the yield for those that were not also selected as either X-ray or mid-IR candidates is low (8\% for "Var. (only)").

Similarly, we can use the narrow variability selection criteria on $A$ and $\gamma$ based on the structure function approach from Schmidt et al. (2010). As shown in Figure 9, these authors used the criteria that $\gamma>0.5 \times \log _{10}(A)+0.50, \gamma>-2 \times$ $\log _{10}(A)-2.25$, and $\gamma>0.055$. For these tighter criteria, $48 \%$ of the variability-selected sources were confirmed to be AGNs 
Table 4

MQS Yields

\begin{tabular}{|c|c|c|c|c|c|c|c|c|}
\hline \multirow[t]{2}{*}{ Selection } & \multicolumn{4}{|c|}{ All MC Sources } & \multicolumn{4}{|c|}{$I<19.5$ mag MC Sources } \\
\hline & Candidates & $\begin{array}{l}\text { Observed } \\
\text { Targets }\end{array}$ & $\begin{array}{c}\text { Confirmed } \\
\text { AGNs }\end{array}$ & $\begin{array}{c}\text { Weighted } \\
\text { Yield } E(\%)\end{array}$ & Candidates & $\begin{array}{l}\text { Observed } \\
\text { Targets }\end{array}$ & $\begin{array}{c}\text { Confirmed } \\
\text { AGNs }\end{array}$ & $\begin{array}{c}\text { Weighted } \\
\text { Yield } E(\%)\end{array}$ \\
\hline Mid-IR QSO-Aa & 2565 & 2127 & 636 & 29 & 969 & 806 & 401 & 49 \\
\hline Mid-IR QSO-Ab & 51 & 36 & 4 & 11 & 13 & 9 & 0 & 0 \\
\hline Mid-IR QSO-Ba & 264 & 219 & 55 & 24 & 86 & 73 & 38 & 51 \\
\hline Mid-IR QSO-Bb & 5 & 2 & 0 & 0 & 1 & 0 & 0 & $\ldots$ \\
\hline Mid-IR YSO-Aa & 131 & 99 & 17 & 15 & 107 & 81 & 14 & 14 \\
\hline Mid-IR YSO-Ab & 45 & 40 & 3 & 7 & 8 & 5 & 0 & 0 \\
\hline Mid-IR YSO-Ba & 24 & 16 & 4 & 27 & 21 & 13 & 3 & 25 \\
\hline Mid-IR YSO-Bb & 20 & 15 & 0 & 0 & 4 & 2 & 0 & 0 \\
\hline Mid-IR (any) & 3106 & 2555 & 721 & 27 & 1210 & 990 & 457 & 44 \\
\hline X-ray (any) & 432 & 299 & 113 & 30 & 283 & 183 & 81 & 33 \\
\hline Var. (any) & 1397 & 1107 & 419 & 34 & 1139 & 862 & 352 & 36 \\
\hline Var (any) + DRW & 650 & 513 & 226 & 45 & 650 & 513 & 226 & 45 \\
\hline X-ray + Mid-IR (any) & 237 & 211 & 105 & 49 & 126 & 112 & 74 & 65 \\
\hline Mid-IR + Var. (any) & 800 & 739 & 384 & 52 & 556 & 504 & 320 & 63 \\
\hline Var. + X-ray (any) & 126 & 112 & 75 & 66 & 104 & 91 & 66 & 71 \\
\hline \multicolumn{9}{|c|}{ Priority 7} \\
\hline Mid-IR (only) & 2173 & 1703 & 300 & 18 & 610 & 451 & 123 & 27 \\
\hline X-ray (only) & 173 & 74 & 2 & 3 & 135 & 57 & 1 & 2 \\
\hline Var. (only) & 574 & 354 & 29 & 8 & 561 & 344 & 26 & 8 \\
\hline \multicolumn{9}{|c|}{ Priority 8} \\
\hline X-ray + Mid-IR (only) & 133 & 113 & 36 & 32 & 44 & 35 & 14 & 40 \\
\hline Mid-IR + Var. (only) & 696 & 641 & 315 & 49 & 474 & 427 & 260 & 61 \\
\hline Var. + X-ray (only) & 22 & 14 & 6 & 43 & 22 & 14 & 6 & 43 \\
\hline \multicolumn{9}{|c|}{ Priority 9} \\
\hline all three & 104 & 98 & 69 & 70 & 82 & 77 & 60 & 78 \\
\hline
\end{tabular}

Note. The weighted yield $E$ is calculated using Equation (1).

and $41 \%(83 \%)$ of the confirmed (and variability-selected) AGNs satisfy the criteria. The $83 \%$ fraction is high because our variability selection method was quite similar to the full procedures from Schmidt et al. (2010). While Schmidt et al. (2010) never contemplated using their method in dense stellar fields, it works reasonably well.

Kim et al. (2012) selected 2566 AGN candidates spread over roughly $40 \mathrm{deg}^{2}$ behind the LMC based on their optical variability in the MACHO survey and then reduced the sample to 663 "high quality" candidates based on their optical, mid-IR, and/or X-ray properties. Although the MACHO sample is brighter, with a median magnitude of $\sim 18.2$ mag rather than our $19.6 \mathrm{mag}$, the Kim et al. (2012) sample has a significantly higher surface density of 31 candidates $\mathrm{deg}^{-2}$ as compared to 13 candidates $\mathrm{deg}^{-2}$ for the MQS sample at the same magnitude limit. For comparison, the expected surface density of quasars brighter than $18.2 \mathrm{mag}$ is only 2.7 quasars $\mathrm{deg}^{-2}$, which means that the contamination levels in the Kim et al. (2012) variability-selected sample are significantly higher than for the MQS samples, with upper limits on the purities of the Kim et al. (2012) and MQS variability-selected samples of order $9 \%$ and $21 \%$, respectively. The surface density of the "high quality" sample is much lower, and in fact drops below the expected surface density of quasars at fainter magnitudes, indicating that it must be substantially incomplete even if it has little contamination. There are 248 (216) matches of the Kim et al. (2012) sample (the high quality subset) with our MQS sample for a matching radius of 3."0 with 133 (131) matches being confirmed quasars. Kim et al. (2012) attempt to compare their selection methods with ours by contrasting the $131 \mathrm{MQS}$ quasars in the sample of 248 candidates matched to their full sample $(61 \%)$ to the $7 \%$ MQS yield (Paper II) for variabilityselected quasars that were neither X-ray nor mid-IR-selected. Even if there was an independent spectroscopic follow-up of the Kim et al. (2012) sample, one would need to either compare samples selected based only on variability $(131 / 216=61 \%$ versus $34 \%$ for the MQS) or variability-selected samples not selected by other methods $(2 / 32=6 \%$ versus $8 \%$ for the MQS), rather than mixing the two possibilities. More fundamentally, unless the Kim et al. (2012) selection methods are completely devoid of any new information on whether sources are quasars, the apparent efficiency of the Kim et al. (2012) sub-sample contained in the MQS sample must be higher than the efficiency of the MQS sample as a whole. In essence, Kim et al. (2012) are adding a fourth selection method and then comparing the completeness of the intersection of (say) selection methods $2+4$ to the completeness of selection method 2 alone. This fact holds true even if both methods are variability selection methods, either independent statistics applied to the same data set or (as in this case) different statistics applied to two different data sets. As we see from Figure 10 and Table 4, the completeness obtained from the intersections of selection methods are always markedly higher than those for one method alone. Without an independent spectroscopic study of the Kim et al. (2012) sample, it is impossible to compare the efficiency of the different selection methods beyond the crude comparison of the surface 


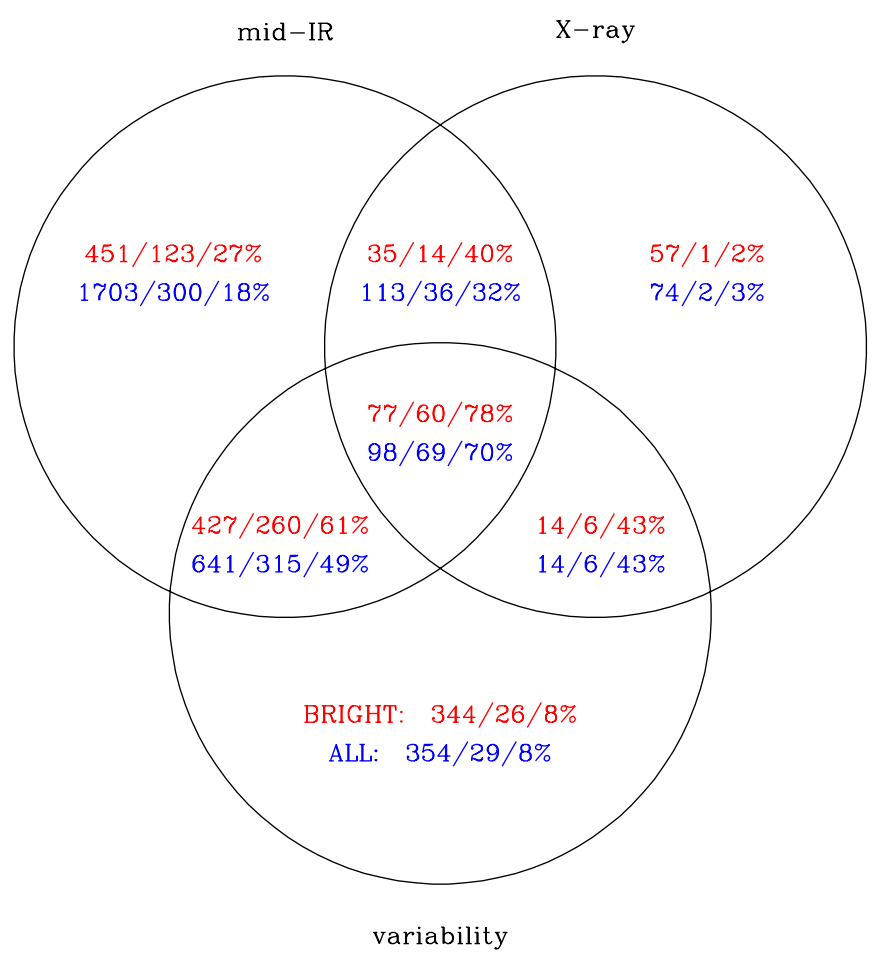

Figure 10. Venn diagram for the confirmed AGNs, showing efficiencies of the three AGN selection methods. The numbers on the left (middle, right) are the numbers of observed targets (confirmed AGNs, yields). The upper (red) numbers are for bright $(I<19.5 \mathrm{mag}$ ) sources and the bottom numbers (blue) are for all sources. See Table 4 for details.

density of candidates to the surface density of quasars discussed above.

Finally, Table 4 shows the effects of using various combinations of the selection methods. For example, samples that combine mid-IR + variability criteria have yields of 52\% $(63 \%)$ for all (bright) sources, samples that combine mid-IR + X-ray criteria have yields of $49 \%(65 \%)$, and samples that apply variability $+\mathrm{X}$-ray criteria have yields of $66 \%$ (71\%). In these results, we include objects independent of their status based on the third selection method. If objects are selected by two methods and not by the third, the yields are generally significantly lower, with $49 \%$ (61\%) for mid-IR + variability all (bright) sources, $32 \%(40 \%)$ for mid-IR + X-ray sources, and $43 \%(43 \%)$ for variability $+\mathrm{X}$-ray sources. The various possible overlapping selection choices are graphically illustrated as a Venn diagram in Figure 10.

\section{SUMMARY}

In this paper, we report the final spectroscopically confirmed AGN sample from the MQS - the largest spectroscopic search for MC quasars to date. We obtained spectra for 2248 (766) LMC (SMC) sources and identified 565 (193) as AGNs. We also confirmed 38 (7) known LMC (SMC) AGNs and were unable to confirm one. The total number of confirmed MQS quasars is 758, of which 713 are new. Thus, the MQS has increased the number of quasars known behind the MCs by an order of magnitude to an overall total of roughly 800 quasars. These sources provide a dense network of proper motion reference points for improving measurements of the internal and bulk proper motions of the MCs (e.g., Kallivayalil et al. 2013; van der Marel \& Kallivayalil 2013) and these are the quasars with the best long-term, densely sampled light curves for studying quasar variability physics (e.g., Kelly et al. 2009; MacLeod et al. 2010). Also, 50 quasars brighter than $I \lesssim 18$ mag enable studies of absorption by the interstellar/intergalactic medium. We roughly estimate that we have achieved $\sim 75 \%$ completeness for $I<19$ mag quasars in the OGLE-III regions of the MCs.

The nature of the AAOmega instrument, with many more fibers than needed given the numbers of quasars brighter than our effective magnitude limit of $I \approx 20.5 \mathrm{mag}$, means that we also obtained spectra of many contaminating LMC sources. Because we only target sources that have "abnormal" properties for stars, the contaminating sources are a mixture of dusty or accreting sources, including many YSOs, PNe, and Be stars. These sources will be discussed elsewhere. Despite fully populating the fibers, the yields from the various selection methods are quite good, particularly when combined. Individual methods typically have yields of order $30 \%$, combinations of two methods have yields of order $55 \%$, and combining all three methods produces a yield of order $70 \%$. Of course, the number of available targets also declines and the overall number of AGNs identified by only one, two, or all three methods is 331,357 , and 69, respectively, because of the usual trade-offs between completeness and contamination. In Kozłowski \& Kochanek (2009) and Kozłowski et al. (2010), we argued that mid-IR and variability selection methods would be effective despite the high stellar densities of the MCs, and the MQS provides excellent confirmation of this statement. Since OGLE-III covered the densest regions of the MCs, expanding the search for quasars to the larger OGLE-IV region will be significantly easier because of the reduced stellar densities. Doing so, however, requires somewhat longer term OGLE-IV light curves to carry out the variability selection since the mid-IR and X-ray surveys of the MCs do not extend over the much larger OGLE-IV survey regions.

We thank the anonymous referee for suggestions that improved the manuscript. This research is based on observations made with the Anglo-Australian Telescope, for which the observing time was granted by the Optical Infrared Coordination Network for Astronomy (OPTICON). This research has made use of the SIMBAD database, operated at CDS, Strasbourg, France. This research has also made use of the NASA/ IPAC Extragalactic Database (NED), which is operated by the Jet Propulsion Laboratory (JPL), California Institute of Technology (Caltech), under contract with the National Aeronautics and Space Administration (NASA). C.S.K. is supported by NSF grant AST-1009756. OGLE is supported by the European Research Council under the European Community's Seventh Framework Programme (FP7/2007-2013), ERC grant agreement no. 246678 to A.U. The work in this paper was partially supported by the Polish Ministry of Science and Higher Education through the program "Ideas Plus" award No. IdP2012 000162 to I.S.

\section{REFERENCES}

Alcock, C., Allsman, R. A., Alves, D. R., et al. 2000, ApJ, 542, 281

Alves, D. R. 2004, NewAR, 48, 659

Alves, D. R., Rejkuba, M., Minniti, D., \& Cook, K. H. 2002, ApJL, 573, L51

Andrae, R., Kim, D.-W., \& Bailer-Jones, C. A. L. 2013, A\&A, 554, A137

Assef, R. J., Kochanek, C. S., Brodwin, M., et al. 2010, ApJ, 713, 970

Bolatto, A. D., Simon, J. D., Stanimirović, S., et al. 2007, ApJ, 655, 212

Bonanos, A. Z., Castro, N., Macri, L. M., \& Kudritzki, R.-P. 2011, ApJL, 729, L9

Butler, N. R., \& Bloom, J. S. 2011, AJ, 141, 93 
Cioni, M.-R. L., van der Marel, R. P., Loup, C., \& Habing, H. J. 2000, A\&A, 359,601

Dobrzycki, A., Eyer, L., Stanek, K. Z., \& Macri, L. M. 2005, A\&A, 442, 495

Dobrzycki, A., Groot, P. J., Macri, L. M., \& Stanek, K. Z. 2002, ApJL, 569, L15

Dobrzycki, A., Macri, L. M., Stanek, K. Z., \& Groot, P. J. 2003a, AJ, 125, 1330

Dobrzycki, A., Stanek, K. Z., Macri, L. M., \& Groot, P. J. 2003b, AJ, 126, 734

Eyer, L. 2002, AcA, 52, 241

Feast, M. 1999, PASP, 111, 775

Geha, M., Alcock, C., Allsman, R. A., et al. 2003, AJ, 125, 1

Gieren, W. P., Fouque, P., \& Gomez, M. 1998, ApJ, 496, 17

Gordon, K. D., Meixner, M., Meade, M. R., et al. 2011, AJ, 142, 102

Groenewegen, M. A. T., \& de Jong, T. 1993, A\&A, 267, 410

Haberl, F., Filipović, M. D., Pietsch, W., \& Kahabka, P. 2000, A\&AS, 142, 41

Haberl, F., \& Pietsch, W. 1999, A\&AS, 139, 277

Haschke, R., Grebel, E. K., \& Duffau, S. 2011, AJ, 141, 158

Hickox, R. C., Jones, C., Forman, W. R., et al. 2009, ApJ, 696, 891

Hojjati, A., Kim, A. G., \& Linder, E. V. 2013, PhRvD, 87, 123512

Humphreys, R. M., \& McElroy, D. B. 1984, ApJ, 284, 565

Ivezic, Z., Tyson, J. A., Acosta, E., et al. 2008, arXiv:0805.2366

Kaiser, N., Aussel, H., Burke, B. E., et al. 2002, Proc. SPIE, 4836, 154

Kallivayalil, N., van der Marel, R. P., Alcock, C., et al. 2006a, ApJ, 638, 772

Kallivayalil, N., van der Marel, R. P., \& Alcock, C. 2006b, ApJ, 652, 1213

Kallivayalil, N., van der Marel, R. P., Besla, G., Anderson, J., \& Alcock, C. 2013, ApJ, 764, 161

Kelly, B. C., Bechtold, J., \& Siemiginowska, A. 2009, ApJ, 698, 895

Kim, D.-W., Protopapas, P., Trichas, M., et al. 2012, ApJ, 747, 107

Kochanek, C. S., Eisenstein, D. J., Cool, R. J., et al. 2012, ApJS, 200, 8

Kozłowski, S., \& Kochanek, C. S. 2009, ApJ, 701, 508

Kozłowski, S., Kochanek, C. S., Jacyszyn, A. M., et al. 2012, ApJ, 746, 27 (Paper II)

Kozłowski, S., Kochanek, C. S., \& Udalski, A. 2011, ApJS, 194, 22 (Paper I)

Kozłowski, S., Kochanek, C. S., Udalski, A., et al. 2010, ApJ, 708, 927

Kozłowski, S., Udalski, A., Wyrzykowski, Ł., et al. 2013, AcA, 63, 1

Lacy, M., Storrie-Lombardi, L. J., Sajina, A., et al. 2004, ApJS, 154, 166

Lane, R. R., Küpper, A. H. W., \& Heggie, D. C. 2012, MNRAS, 423, 2845

Lewis, I. J., Cannon, R. D., Taylor, K., et al. 2002, MNRAS, 333, 279

MacLeod, C. L., Brooks, K., Ivezić, Ž., et al. 2011, ApJ, 728, 26

MacLeod, C. L., Ivezić, Ž., Kochanek, C. S., et al. 2010, ApJ, 721, 1014

MacLeod, C. L., Ivezić, Ž., Sesar, B., et al. 2012, ApJ, 753, 106

Massey, P., Lang, C. C., Degioia-Eastwood, K., \& Garmany, C. D. 1995, ApJ, 438, 188

Meixner, M., Gordon, K. D., Indebetouw, R., et al. 2006, AJ, 132, 2268
Miszalski, B., Shortridge, K., Saunders, W., Parker, Q. A., \& Croom, S. M. 2006, MNRAS, 371, 1537

Mushotzky, R. F., Edelson, R., Baumgartner, W., \& Gandhi, P. 2011, ApJL, 743, L12

Palanque-Delabrouille, N., Yeche, C., Myers, A. D., et al. 2011, A\&A, 530, A122

Piatek, S., Pryor, C., \& Olszewski, E. W. 2008, AJ, 135, 1024

Pichara, K., Protopapas, P., Kim, D.-W., Marquette, J.-B., \& Tisserand, P. 2012, MNRAS, 427, 1284

Pietrzyński, G., Graczyk, D., Gieren, W., et al. 2013, Natur, 495, 76

Press, W. H., Rybicki, G. B., \& Hewitt, J. N. 1992, ApJ, 385, 404

Richards, G. T., Strauss, M. A., Fan, X., et al. 2006, AJ, 131, 2766

Rybicki, G. B., \& Press, W. H. 1992, ApJ, 398, 169

Rybicki, G. B., \& Press, W. H. 1995, PhRvL, 74, 1060

Schmidt, K. B., Marshall, P. J., Rix, H.-W., et al. 2010, ApJ, 714, 1194

Schmidtke, P. C., Cowley, A. P., Frattare, L. M., et al. 1994, PASP, 106, 843

Schommer, R. A., Olszewski, E. W., \& Aaronson, M. 1984, ApJL, 285, L53

Sharp, R., Saunders, W., Smith, G., et al. 2006, Proc. SPIE, 6269, 62690G

Soszyński, I., Poleski, R., Udalski, A., et al. 2008, AcA, 58, 163

Soszyński, I., Poleski, R., Udalski, A., et al. 2010, AcA, 60, 17

Soszyński, I., Udalski, A., Szymański, M. K., et al. 2009a, AcA, 59, 1

Soszyński, I., Udalski, A., Szymański, M. K., et al. 2009b, AcA, 59, 239

Stern, D., Eisenhardt, P., Gorjian, V., et al. 2005, ApJ, 631, 163

Taylor, K., Bailey, J., Wilkins, T., Shortridge, K., \& Glazebrook, K. 1996, in ASP Conf. Ser. 101, Astronomical Data Analysis Software and Systems V, ed. G. H. Jacoby \& J. Barnes (San Francisco, CA: ASP), 195

Tisserand, P., Le Guillou, L., Afonso, C., et al. 2007, A\&A, 469, 387

Udalski, A. 2000a, AcA, 50, 279

Udalski, A. 2000b, ApJL, 531, L25

Udalski, A., Kubiak, M., \& Szymanski, M. 1997, AcA, 47, 319

Udalski, A., Soszynski, I., Szymanski, M. K., et al. 2008a, AcA, 58, 89

Udalski, A., Soszyński, I., Szymański, M. K., et al. 2008b, AcA, 58, 329

Udalski, A., Szymański, M. K., Soszyński, I., \& Poleski, R. 2008c, AcA, 58,69

Vanden Berk, D. E., Richards, G. T., Bauer, A., et al. 2001, AJ, 122, 549

van der Marel, R. P., Alves, D. R., Hardy, E., \& Suntzeff, N. B. 2002, AJ, 124, 2639

van der Marel, R. P., \& Kallivayalil, N. 2013, arXiv:1305.4641

Weingartner, J. C., \& Draine, B. T. 2001, ApJ, 548, 296

Werner, M. W., Roellig, T. L., Low, F. J., et al. 2004, ApJS, 154, 1

Wyrzykowski, L., Skowron, J., Kozłowski, S., et al. 2011, MNRAS, 416, 2949

Zu, Y., Kochanek, C. S., Kozłowski, S., \& Udalski, A. 2013, ApJ, 765, 106

Zu, Y., Kochanek, C. S., \& Peterson, B. M. 2011, ApJ, 735, 80 